\title{
Sturm-Liouville problem with moving discontinuity points
}

\author{
Fatma Hıra ${ }^{1 *}$ and Nihat Altınışık ${ }^{2}$
}

\section{*Correspondence:}

fatma.hira@omu.edu.tr

'Department of Mathematics,

Faculty of Arts and Sciences, Hitit

University, Çorum, 19030, Turkey

Full list of author information is

available at the end of the article
(C) 2015 Hıra and Altınısıı. This article is distributed under the terms of the Creative Commons Attribution 4.0 International License (http://creativecommons.org/licenses/by/4.0/), which permits unrestricted use, distribution, and reproduction in any medium, provided you give appropriate credit to the original author(s) and the source, provide a link to the Creative Commons license, and indicate if changes were made.

\begin{abstract}
In this paper, we present a new discontinuous Sturm-Liouville problem with symmetrically located discontinuities which are defined depending on a parameter in the neighborhood of an interior point in the interval. Also the problem contains an eigenparameter in a boundary condition. We investigate some spectral properties of the eigenvalues, obtain asymptotic formulae for the eigenvalues and the corresponding eigenfunctions and construct Green's function for the problem. We give an illustrative example with tables and figures at the end of the paper.
\end{abstract}

MSC: 34B09; 34L10; 34B24; 34B27

Keywords: Sturm-Liouville problem; moving discontinuity point; eigenvalue and eigenfunction; Green's function

\section{Introduction}

The Sturm-Liouville theory plays an important role in solving many mathematical physics problems [1]. Such research is motivated by the theory of heat and mass transfer, or vibrating string problems when the string is loaded additionally with point masses (see [2, 3]; see also the references therein). When using Fourier's method for heat transfer problems, it becomes necessary to solve a related Sturm-Liouville problem in which the separation constant plays the role of a spectral parameter (or eigenparameter). In the case of inhomogeneous materials and composite walls, the Sturm-Liouville equation has variable and not necessarily continuous coefficients, so that transmission conditions across the interfaces should be added in the problem. Heat conduction problems in composite walls have been analysed by several researchers [4-6]. In all of these works, the temperature distribution of composite walls involving two or more layers are investigated spectrally and the results are formulated by the eigenvalues and eigenfunctions of the auxiliary spectral problem. In [7], the presence of an infinite number of eigenvalues has been shown and an asymptotic formula has been obtained for the eigenvalues of the spectral problem for the temperature distribution in composite walls. The problem in that work included a two-layer composite wall consisting of different materials, having a common contact surface. Physically, we can say that the problem in the present paper is derived from a heat conduction problem of a three-layer composite wall consisting of two interfaces that are located symmetrically. Thus we add transmission conditions at these interfaces i.e. at the points of discontinuity to the problem. 
Eigenvalue problems have been studied by some authors in the continuous case; see [810]. In recent years, there were also some studies on the discontinuous eigenvalue problems [11-19] and construction of Green's functions for discontinuous Sturm-Liouville problems which contain an eigenparameter under one or two boundary conditions $[14,16]$.

We consider the following Sturm-Liouville problem:

$$
\tau(y):=-y \prime \prime+q(x) y=\lambda y, \quad x \in I,
$$

with an eigenparameter dependent on a boundary condition:

$$
\begin{aligned}
& B_{a}(y):=\beta_{1} y(a)+\beta_{2} y^{\prime}(a)=0, \\
& B_{b}(y):=\lambda\left(\alpha_{1}^{\prime} y(b)-\alpha_{2}^{\prime} y^{\prime}(b)\right)+\left(\alpha_{1} y(b)-\alpha_{2} y^{\prime}(b)\right)=0,
\end{aligned}
$$

and coupled transmission conditions at the points of discontinuity, $\theta_{-\varepsilon}$ and $\theta_{+\varepsilon}$ :

$$
\begin{aligned}
& T_{-\varepsilon}(y):=y\left(\theta_{-\varepsilon}+\right)-\left(\mu_{1} y\left(\theta_{-\varepsilon}-\right)+\mu_{2} y^{\prime}\left(\theta_{-\varepsilon}-\right)\right)=0, \\
& T_{-\varepsilon}^{\prime}(y):=y^{\prime}\left(\theta_{-\varepsilon}+\right)-\left(\mu_{1}^{\prime} y\left(\theta_{-\varepsilon}-\right)+\mu_{2}^{\prime} y^{\prime}\left(\theta_{-\varepsilon}-\right)\right)=0, \\
& T_{+\varepsilon}(y):=y\left(\theta_{+\varepsilon}+\right)-\left(\eta_{1} y\left(\theta_{+\varepsilon}-\right)+\eta_{2} y^{\prime}\left(\theta_{+\varepsilon}-\right)\right)=0, \\
& T_{+\varepsilon}^{\prime}(y):=y^{\prime}\left(\theta_{+\varepsilon}+\right)-\left(\eta_{1}^{\prime} y\left(\theta_{+\varepsilon}-\right)+\eta_{2}^{\prime} y^{\prime}\left(\theta_{+\varepsilon}-\right)\right)=0,
\end{aligned}
$$

where $I:=\left[a, \theta_{-\varepsilon}\right) \cup\left(\theta_{-\varepsilon}, \theta_{+\varepsilon}\right) \cup\left(\theta_{+\varepsilon}, b\right] ; \theta \in(a, b)$ and

$$
0<\varepsilon<\min \{\theta-a, b-\theta\}
$$

$\lambda$ is a spectral parameter; $q(x)$ is a given real valued function which is continuous in $\left[a, \theta_{-\varepsilon}\right)$, $\left(\theta_{-\varepsilon}, \theta_{+\varepsilon}\right)$ and $\left(\theta_{+\varepsilon}, b\right]$ and has finite limits $q\left(\theta_{-\varepsilon} \pm\right), q\left(\theta_{+\varepsilon} \pm\right) ; \beta_{i}, \alpha_{i}, \alpha_{i}^{\prime}, \mu_{i}, \mu_{i}^{\prime}, \eta_{i}, \eta_{i}^{\prime}(i=1,2)$ are real numbers such that $\left|\beta_{1}\right|+\left|\beta_{2}\right| \neq 0$ and

$$
\begin{aligned}
& \rho:=\operatorname{det}\left(\begin{array}{ll}
\alpha_{1}^{\prime} & \alpha_{2}^{\prime} \\
\alpha_{1} & \alpha_{2}
\end{array}\right)>0, \\
& D_{1}:=\operatorname{det}\left(\begin{array}{ll}
\mu_{1} & \mu_{2} \\
\mu_{1}^{\prime} & \mu_{2}^{\prime}
\end{array}\right)>0, \quad D_{2}:=\operatorname{det}\left(\begin{array}{ll}
\eta_{1} & \eta_{2} \\
\eta_{1}^{\prime} & \eta_{2}^{\prime}
\end{array}\right)>0 .
\end{aligned}
$$

Also for convenience we will use the notations $\theta_{ \pm \varepsilon} \pm:=(\theta \pm \varepsilon) \pm 0$.

We introduce a new Sturm-Liouville problem with discontinuities which are defined depending on a parameter in the neighborhood of an interior point of $\theta . \varepsilon$ is a parameter controlling the variation of the neighborhood process and by using the variation of this parameter, it is possible to determine points of discontinuity. Thus, the points of discontinuity can be placed anywhere. Accordingly, they can be called 'moving discontinuity points' In the special case, when our problem is not with an eigenparameter in the boundary condition and discontinuities are $d_{1}=d$ and $d_{2}=\pi-d$ in the interval $[0, \pi]$, was derived in $[11,12]$. 
The main aim of the present work is to present moving discontinuity points, to emphasise some of the distinguishing properties of such problems from the ones of discontinuous Sturm-Liouville problems, and to study the spectral properties of the problem. To achieve our aim we extend some classic results of Sturm-Liouville theory to the new moving discontinuous case. First, we define a linear operator $A$ in a suitable Hilbert space $H$ such that the eigenvalues of the problem (1)-(7) coincide with those of $A$ and construct a special fundamental system of solutions. Then we obtain the asymptotic formulae for the eigenvalues and the corresponding eigenfunctions depending on the parameter $\varepsilon$ and construct Green's function for the problem (1)-(7). Finally, an illustrative example, which shows how to determine discontinuities for different values of $\varepsilon$, is given.

\section{An operator formulation}

In this section we will introduce the special inner product in the Hilbert space $L_{2}(a, b) \oplus \mathbb{C}$ and a symmetric linear operator $A$ is defined in this Hilbert space in such a way that the problem (1)-(7) can be considered as the eigenvalue problem of this operator.

Definition 1 We define a Hilbert space $H$ of two component vectors by $H:=L_{2}(a, b) \oplus \mathbb{C}$ with the inner product:

$$
\begin{aligned}
\langle U, V\rangle_{H}:= & \int_{a}^{\theta_{-\varepsilon}} u(x) \bar{v}(x) d x+\frac{1}{D_{1}} \int_{\theta_{-\varepsilon}}^{\theta_{+\varepsilon}} u(x) \bar{v}(x) d x+\frac{1}{D_{1} D_{2}} \int_{\theta_{+\varepsilon}}^{b} u(x) \bar{v}(x) d x \\
& +\frac{1}{\rho D_{1} D_{2}} h \bar{k}
\end{aligned}
$$

where $U=\left(\begin{array}{c}u(x) \\ h\end{array}\right), V=\left(\begin{array}{c}v(x) \\ k\end{array}\right) \in H, u(x), v(x) \in L_{2}(a, b)$ and $h, k \in \mathbb{C}$.

For a function $u(x)$, which is defined on $I$ and has finite limits $u\left(\theta_{-\varepsilon} \pm\right):=\lim _{x \rightarrow \theta_{-\varepsilon} \pm} u(x)$, $u\left(\theta_{+\varepsilon} \pm\right):=\lim _{x \rightarrow \theta_{+\varepsilon} \pm} u(x)$, by $u_{(i)}(x)(i=\overline{1,4})$ we denote the function

$$
\begin{aligned}
& u_{(1)}(x):=\left\{\begin{array}{ll}
u(x), & x \in\left[a, \theta_{-\varepsilon}\right), \\
u\left(\theta_{-\varepsilon}-\right), & x=\theta_{-\varepsilon},
\end{array} \quad u_{(2)}(x):= \begin{cases}u\left(\theta_{-\varepsilon}+\right), & x=\theta_{-\varepsilon}, \\
u(x), & x \in\left(\theta_{-\varepsilon}, \theta_{+\varepsilon}\right),\end{cases} \right. \\
& u_{(3)}(x):=\left\{\begin{array}{ll}
u(x), & x \in\left(\theta_{-\varepsilon}, \theta_{+\varepsilon}\right), \\
u\left(\theta_{+\varepsilon}\right), & x=\theta_{+\varepsilon},
\end{array} \quad u_{(4)}(x):= \begin{cases}u\left(\theta_{+\varepsilon}+\right), & x=\theta_{+\varepsilon}, \\
u(x), & x \in\left(\theta_{+\varepsilon}, b\right],\end{cases} \right.
\end{aligned}
$$

which are defined on $I_{1}=\left[a, \theta_{-\varepsilon}\right], I_{2}=\left[\theta_{-\varepsilon}, \theta_{+\varepsilon}\right]$ and $I_{3}=\left[\theta_{+\varepsilon}, b\right]$, respectively.

For convenience we will use the notations

$$
R(u):=\alpha_{1} u(b)-\alpha_{2} u^{\prime}(b), \quad R^{\prime}(u):=\alpha_{1}^{\prime} u(b)-\alpha_{2}^{\prime} u^{\prime}(b) .
$$

Definition 2 We define a linear operator $A: D(A) \rightarrow H$ by

$$
A\left(\begin{array}{c}
u(x) \\
R^{\prime}(u)
\end{array}\right):=\left(\begin{array}{c}
\tau(u) \\
-R(u)
\end{array}\right),
$$

where the domain $D(A)$ of the linear operator $A$ is defined as the set of all $U=\left(\begin{array}{c}u(x) \\ R^{\prime}(u)\end{array}\right)$ which satisfies the conditions (i) $\tau(u) \in L_{2}(a, b)$, (ii) $u_{(i)}(\cdot), u_{(i)}^{\prime}(\cdot)$ are absolutely continuous functions in $I_{i}(i=1,2,3)$, (iii) $B_{a}(u)=0$, (iv) $T_{ \pm \varepsilon}(u)=T_{ \pm \varepsilon}^{\prime}(u)=0$. 
Now we can rewrite the problem (1)-(7) in the operator form as $A U=\lambda U$ where $U=$ $\left(\begin{array}{c}u(x) \\ R^{\prime}(u)\end{array}\right) \in D(A)$.

The eigenvalues and eigenfunctions of the problem (1)-(7) are defined as the eigenvalues and the first components of the corresponding eigenelements of the operator $A$, respectively.

Lemma 1 The operator $A$ in $H$ is symmetric.

Proof For $U, V \in D(A)$,

$$
\begin{aligned}
\langle A U, V\rangle_{H}= & \int_{a}^{\theta_{-\varepsilon}} \tau(u) \bar{v}(x) d x+\frac{1}{D_{1}} \int_{\theta_{-\varepsilon}}^{\theta_{+\varepsilon}} \tau(u) \bar{v}(x) d x+\frac{1}{D_{1} D_{2}} \int_{\theta_{+\varepsilon}}^{b} \tau(u) \bar{v}(x) d x \\
& -\frac{1}{\rho D_{1} D_{2}} R(u) R^{\prime}(\bar{v}) .
\end{aligned}
$$

By two partial integrations, we obtain

$$
\begin{aligned}
\langle A U, V\rangle_{H}= & \langle U, A V\rangle_{H}+W\left(u, \bar{v} ; \theta_{-\varepsilon}-\right)-W(u, \bar{v} ; a)+\frac{1}{D_{1}} W\left(u, \bar{v} ; \theta_{+\varepsilon}-\right) \\
& -\frac{1}{D_{1}} W\left(u, \bar{v} ; \theta_{-\varepsilon}+\right)+\frac{1}{D_{1} D_{2}} W(u, \bar{v} ; b)-\frac{1}{D_{1} D_{2}} W\left(u, \bar{v} ; \theta_{+\varepsilon}+\right) \\
& -\frac{1}{\rho D_{1} D_{2}}\left(R(u) R^{\prime}(\bar{v})-R^{\prime}(u) R(\bar{v})\right)
\end{aligned}
$$

where, as usual, by $W(u, v ; x)$ we denote the Wronskian of the functions $u(x)$ and $v(x)$ :

$$
W(u, v ; x)=u(x) v^{\prime}(x)-u^{\prime}(x) v(x)
$$

Since $u$ and $\bar{v}$ satisfy (2), it follows that

$$
W(u, \bar{v} ; a)=0,
$$

from (4)-(7), we get

$$
\begin{aligned}
& W\left(u, \bar{v} ; \theta_{-\varepsilon}+\right)=D_{1} W\left(u, \bar{v} ; \theta_{-\varepsilon}-\right), \\
& W\left(u, \bar{v} ; \theta_{+\varepsilon}+\right)=D_{2} W\left(u, \bar{v} ; \theta_{+\varepsilon}-\right) .
\end{aligned}
$$

Further, from (11) it is easy to verify that

$$
R(u) R^{\prime}(\bar{v})-R^{\prime}(u) R(\bar{v})=\rho W(u, \bar{v} ; b) .
$$

Finally, substituting (15)-(18) into (14), we have

$$
\langle A U, V\rangle_{H}=\langle U, A V\rangle_{H},
$$

so $A$ is symmetric.

Corollary 1 All eigenvalues of the problem (1)-(7) are real. 


\section{Construction of fundamental solutions}

We will define the two solutions

$$
\phi_{\lambda}(x)=\left\{\begin{array}{ll}
\phi_{-\varepsilon, \lambda}(x), & x \in\left[a, \theta_{-\varepsilon}\right), \\
\phi_{\varepsilon, \lambda}(x), & x \in\left(\theta_{-\varepsilon}, \theta_{+\varepsilon}\right), \\
\phi_{+\varepsilon, \lambda}(x), & x \in\left(\theta_{+\varepsilon}, b\right],
\end{array} \quad \chi_{\lambda}(x)= \begin{cases}\chi_{-\varepsilon, \lambda}(x), & x \in\left[a, \theta_{-\varepsilon}\right), \\
\chi_{\varepsilon, \lambda}(x), & x \in\left(\theta_{-\varepsilon}, \theta_{+\varepsilon}\right), \\
\chi_{+\varepsilon, \lambda}(x), & x \in\left(\theta_{+\varepsilon}, b\right]\end{cases}\right.
$$

of (1) as follows: Let $\phi_{-\varepsilon, \lambda}(x)=\phi_{-\varepsilon}(x, \lambda)$ be the solution of $(1)$ on $\left[a, \theta_{-\varepsilon}\right]$, which satisfies the initial conditions

$$
y(a)=\beta_{2}, \quad y^{\prime}(a)=-\beta_{1} .
$$

By virtue of Theorem 1.5 in [20], after defining this solution, we define the solution $\phi_{\varepsilon, \lambda}(x)=\phi_{\varepsilon}(x, \lambda)$ of $(1)$ on $\left[\theta_{-\varepsilon}, \theta_{+\varepsilon}\right]$ by means of the solution $\phi_{-\varepsilon, \lambda}(x)$ by the nonstandard initial conditions

$$
\begin{aligned}
& y\left(\theta_{-\varepsilon}+\right)=\mu_{1} \phi_{-\varepsilon, \lambda}\left(\theta_{-\varepsilon}-\right)+\mu_{2} \phi_{-\varepsilon, \lambda}^{\prime}\left(\theta_{-\varepsilon}-\right), \\
& y^{\prime}\left(\theta_{-\varepsilon}+\right)=\mu_{1}^{\prime} \phi_{-\varepsilon, \lambda}\left(\theta_{-\varepsilon}-\right)+\mu_{2}^{\prime} \phi_{-\varepsilon, \lambda}^{\prime}\left(\theta_{-\varepsilon}-\right) .
\end{aligned}
$$

After defining this solution, we may define the solution $\phi_{+\varepsilon, \lambda}(x)=\phi_{+\varepsilon}(x, \lambda)$ of (1) on $\left[\theta_{+\varepsilon}, b\right]$ by means of the solution $\phi_{\varepsilon, \lambda}(x)$ by the nonstandard initial conditions

$$
\begin{aligned}
& y\left(\theta_{+\varepsilon}+\right)=\eta_{1} \phi_{\varepsilon, \lambda}\left(\theta_{+\varepsilon}-\right)+\eta_{2} \phi_{\varepsilon, \lambda}^{\prime}\left(\theta_{+\varepsilon}-\right), \\
& y^{\prime}\left(\theta_{+\varepsilon}+\right)=\eta_{1}^{\prime} \phi_{\varepsilon, \lambda}\left(\theta_{+\varepsilon}-\right)+\eta_{2}^{\prime} \phi_{\varepsilon, \lambda}^{\prime}\left(\theta_{+\varepsilon}-\right) .
\end{aligned}
$$

Hence, $\phi_{\lambda}(x)=\phi(x, \lambda)$ satisfies of (1), (2) and (4)-(7) on $I$.

Analogously, first we define the solution $\chi_{+\varepsilon, \lambda}(x)=\chi_{+\varepsilon}(x, \lambda)$ on $\left[\theta_{+\varepsilon}, b\right]$ by the initial conditions

$$
y(b)=\alpha_{2}^{\prime} \lambda+\alpha_{2}, \quad y^{\prime}(b)=\alpha_{1}^{\prime} \lambda+\alpha_{1} .
$$

Again, after defining this solution, we define the solution $\chi_{\varepsilon, \lambda}(x)=\chi_{\varepsilon}(x, \lambda)$ of $(1)$ on $\left[\theta_{-\varepsilon}, \theta_{+\varepsilon}\right]$ by the initial conditions

$$
\begin{aligned}
& y\left(\theta_{+\varepsilon}-\right)=\frac{1}{D_{2}}\left(\eta_{2}^{\prime} \chi_{+\varepsilon, \lambda}\left(\theta_{+\varepsilon}+\right)-\eta_{2} \chi_{+\varepsilon, \lambda}^{\prime}\left(\theta_{+\varepsilon}+\right)\right), \\
& y^{\prime}\left(\theta_{+\varepsilon}-\right)=\frac{1}{D_{2}}\left(-\eta_{1}^{\prime} \chi_{+\varepsilon, \lambda}\left(\theta_{+\varepsilon}+\right)+\eta_{1} \chi_{+\varepsilon, \lambda}^{\prime}\left(\theta_{+\varepsilon}+\right)\right) .
\end{aligned}
$$

After defining this solution, we define the solution $\chi_{-\varepsilon, \lambda}(x)=\chi_{-\varepsilon}(x, \lambda)$ of $(1)$ on $\left[a, \theta_{-\varepsilon}\right]$ by the initial conditions

$$
\begin{aligned}
& y\left(\theta_{-\varepsilon}-\right)=\frac{1}{D_{1}}\left(\mu_{2}^{\prime} \chi_{\varepsilon, \lambda}\left(\theta_{-\varepsilon}+\right)-\mu_{2} \chi_{\varepsilon, \lambda}^{\prime}\left(\theta_{-\varepsilon}+\right)\right), \\
& y^{\prime}\left(\theta_{-\varepsilon}-\right)=\frac{1}{D_{1}}\left(-\mu_{1}^{\prime} \chi_{\varepsilon, \lambda}\left(\theta_{-\varepsilon}+\right)+\mu_{1} \chi_{\varepsilon, \lambda}^{\prime}\left(\theta_{-\varepsilon}+\right)\right) .
\end{aligned}
$$

Hence, $\chi_{\lambda}(x)=\chi(x, \lambda)$ satisfies of (1), (3) and (4)-(7) on $I$. 
Let us consider the Wronskians

$$
\begin{aligned}
& W\left(\phi_{ \pm \varepsilon, \lambda}, \chi_{ \pm \varepsilon, \lambda} ; x\right)=\phi_{ \pm \varepsilon, \lambda}(x) \chi_{ \pm \varepsilon, \lambda}^{\prime}(x)-\phi_{ \pm \varepsilon, \lambda}^{\prime}(x) \chi_{ \pm \varepsilon, \lambda}(x), \\
& W\left(\phi_{\varepsilon, \lambda}, \chi_{\varepsilon, \lambda} ; x\right)=\phi_{\varepsilon, \lambda}(x) \chi_{\varepsilon, \lambda}^{\prime}(x)-\phi_{\varepsilon, \lambda}^{\prime}(x) \chi_{\varepsilon, \lambda}(x),
\end{aligned}
$$

which are independent of $x \in I_{i}(i=1,2,3)$ and entire functions where $I_{i}(i=1,2,3)$. After a short calculation we see that $D_{1} D_{2} \omega_{-\varepsilon}(\lambda)=D_{2} \omega_{\varepsilon}(\lambda)=\omega_{+\varepsilon}(\lambda)$. Now we may introduce the characteristic function $\omega(\lambda)$ as

$$
\omega(\lambda):=\omega_{-\varepsilon}(\lambda)=\frac{1}{D_{1}} \omega_{\varepsilon}(\lambda)=\frac{1}{D_{1} D_{2}} \omega_{+\varepsilon}(\lambda)
$$

Corollary 2 The zeros of the functions $\omega_{-\varepsilon}(\lambda), \omega_{\varepsilon}(\lambda)$ and $\omega_{+\varepsilon}(\lambda)$ coincide.

Theorem 1 The eigenvalues of the problem (1)-(7) are the zeros of the function $\omega(\lambda)$.

Proof Let $\omega\left(\lambda_{0}\right)=0$. Then $W\left(\phi_{-\varepsilon, \lambda_{0}}, \chi_{-\varepsilon, \lambda_{0}} ; x\right)=0$ and therefore the functions $\phi_{-\varepsilon, \lambda_{0}}(x)$ and $\chi_{-\varepsilon, \lambda_{0}}(x)$ are linearly dependent, i.e. $\chi_{-\varepsilon, \lambda_{0}}(x)=k_{1} \phi_{-\varepsilon, \lambda_{0}}(x), x \in\left[a, \theta_{-\varepsilon}\right]$ for some $k_{1} \neq 0$. From this, it follows that $\chi_{\lambda_{0}}(x)$ satisfies also the first boundary condition (2), so $\chi_{\lambda_{0}}(x)$ is an eigenfunction for the eigenvalue $\lambda_{0}$.

Now let $y\left(x, \lambda_{0}\right)$ be any eigenfunction correspond to eigenvalue $\lambda_{0}$, but $\omega\left(\lambda_{0}\right) \neq 0$. Then the pair of the functions $\left(\phi_{-\varepsilon}(\cdot), \chi_{-\varepsilon}(\cdot)\right),\left(\phi_{\varepsilon}(\cdot), \chi_{\varepsilon}(\cdot)\right)$ and $\left(\phi_{+\varepsilon}(\cdot), \chi_{+\varepsilon}(\cdot)\right)$ would be linearly independent on $I_{i}(i=1,2,3)$, respectively. Therefore $y\left(x, \lambda_{0}\right)$ may be represented as

$$
y\left(x, \lambda_{0}\right)= \begin{cases}c_{1} \phi_{-\varepsilon, \lambda_{0}}(x)+c_{2} \chi_{-\varepsilon, \lambda_{0}}(x), & x \in\left[a, \theta_{-\varepsilon}\right), \\ c_{3} \phi_{\varepsilon, \lambda_{0}}(x)+c_{4} \chi_{\varepsilon, \lambda_{0}}(x), & x \in\left(\theta_{-\varepsilon}, \theta_{+\varepsilon}\right), \\ c_{5} \phi_{+\varepsilon, \lambda_{0}}(x)+c_{6} \chi_{+\varepsilon, \lambda_{0}}(x), & x \in\left(\theta_{+\varepsilon}, b\right],\end{cases}
$$

where at least one of the constants $c_{i}(i=\overline{1,6})$ is not zero. Considering the equations

$$
\begin{aligned}
& B_{a}\left(y\left(x, \lambda_{0}\right)\right)=0, \quad B_{b}\left(y\left(x, \lambda_{0}\right)\right)=0, \\
& T_{ \pm \varepsilon}\left(y\left(x, \lambda_{0}\right)\right)=0, \quad T_{ \pm \varepsilon}^{\prime}\left(y\left(x, \lambda_{0}\right)\right)=0,
\end{aligned}
$$

as a system of linear equations of the variables $c_{i}(i=\overline{1,6})$ and taking (21), (22), (24) and (25) into account, it follows that the determinant of this system is

$$
\begin{aligned}
& \left|\begin{array}{cccccc}
0 & \omega_{-\varepsilon}\left(\lambda_{0}\right) & 0 & 0 & 0 & 0 \\
\phi_{\varepsilon, \lambda_{0}}\left(\theta_{-\varepsilon}+\right) & \chi_{\varepsilon, \lambda_{0}}\left(\theta_{-\varepsilon}+\right) & -\phi_{\varepsilon, \lambda_{0}}\left(\theta_{-\varepsilon}+\right) & -\chi_{\varepsilon, \lambda_{0}}\left(\theta_{-\varepsilon}+\right) & 0 & 0 \\
\phi_{\varepsilon, \lambda_{0}}^{\prime}\left(\theta_{-\varepsilon}+\right) & \chi_{\varepsilon, \lambda_{0}}^{\prime}\left(\theta_{-\varepsilon}+\right) & -\phi_{\varepsilon, \lambda_{0}}^{\prime}\left(\theta_{-\varepsilon}+\right) & -\chi_{\varepsilon, \lambda_{0}}^{\prime}\left(\theta_{-\varepsilon}+\right) & 0 & 0 \\
0 & 0 & \phi_{+\varepsilon, \lambda_{0}}\left(\theta_{+\varepsilon}+\right) & \chi_{+\varepsilon, \lambda_{0}}\left(\theta_{+\varepsilon}+\right) & -\phi_{+\varepsilon, \lambda_{0}}\left(\theta_{+\varepsilon}+\right) & -\chi_{+\varepsilon, \lambda_{0}}\left(\theta_{+\varepsilon}+\right) \\
0 & 0 & \phi_{+\varepsilon, \lambda_{0}}^{\prime}\left(\theta_{+\varepsilon}+\right) & \chi_{+\varepsilon, \lambda_{0}}^{\prime}\left(\theta_{+\varepsilon}+\right) & -\phi_{+\varepsilon, \lambda_{0}}^{\prime}\left(\theta_{+\varepsilon}+\right) & -\chi_{+\varepsilon, \lambda_{0}}^{\prime}\left(\theta_{+\varepsilon}+\right) \\
0 & 0 & 0 & 0 & \omega_{+\varepsilon}\left(\lambda_{0}\right) & 0
\end{array}\right| \\
& =-\omega_{-\varepsilon}\left(\lambda_{0}\right) \omega_{\varepsilon}\left(\lambda_{0}\right) \omega_{+\varepsilon}^{2}\left(\lambda_{0}\right) \neq 0 .
\end{aligned}
$$

Therefore, the system has only the trivial solution $c_{i}=0(i=\overline{1,6})$. Thus we get a contradiction, which completes the proof. 


\section{Asymptotic approximate formulae}

Now we will derive asymptotic formulae of the eigenvalues and eigenfunctions in a way similar to the techniques of $[9,20]$ and $[13,15]$.

Lemma 2 Let $\phi_{\lambda}(x)$ be the solution of equation (1) defined in Section 3, and let $\lambda=s^{2}$. Then the following integral equations hold for $k=0$ and $k=1$ :

$$
\begin{aligned}
\phi_{-\varepsilon, \lambda}^{(k)}(x)= & \beta_{2}(\cos (s(x-a)))^{(k)}-\frac{\beta_{1}}{s}(\sin (s(x-a)))^{(k)} \\
& +\frac{1}{s} \int_{a}^{x} \sin (s(x-t))^{(k)} q(t) \phi_{-\varepsilon, \lambda}(t) d t, \\
\phi_{\varepsilon, \lambda}^{(k)}(x)= & \left(\mu_{1} \phi_{-\varepsilon, \lambda}\left(\theta_{-\varepsilon}-\right)+\mu_{2} \phi_{-\varepsilon, \lambda}^{\prime}\left(\theta_{-\varepsilon}-\right)\right)\left(\cos \left(s\left(x-\theta_{-\varepsilon}\right)\right)\right)^{(k)} \\
& +\frac{1}{s}\left(\mu_{1}^{\prime} \phi_{-\varepsilon, \lambda}\left(\theta_{-\varepsilon}-\right)+\mu_{2}^{\prime} \phi_{-\varepsilon, \lambda}^{\prime}\left(\theta_{-\varepsilon}-\right)\right)\left(\sin \left(s\left(x-\theta_{-\varepsilon}\right)\right)\right)^{(k)} \\
& +\frac{1}{s} \int_{\theta_{-\varepsilon}}^{x}(\sin (s(x-t)))^{(k)} q(t) \phi_{\varepsilon, \lambda}(t) d t, \\
\phi_{+\varepsilon, \lambda}^{(k)}(x)= & \left(\eta_{1} \phi_{\varepsilon, \lambda}\left(\theta_{+\varepsilon}-\right)+\eta_{2} \phi_{\varepsilon, \lambda}^{\prime}\left(\theta_{+\varepsilon}-\right)\right)\left(\cos \left(s\left(x-\theta_{+\varepsilon}\right)\right)\right)^{(k)} \\
& +\frac{1}{s}\left(\eta_{1}^{\prime} \phi_{\varepsilon, \lambda}\left(\theta_{+\varepsilon}-\right)+\eta_{2}^{\prime} \phi_{\varepsilon, \lambda}^{\prime}\left(\theta_{+\varepsilon}-\right)\right)\left(\sin \left(s\left(x-\theta_{+\varepsilon}\right)\right)\right)^{(k)} \\
& +\frac{1}{s} \int_{\theta_{+\varepsilon}}^{x}(\sin (s(x-t)))^{(k)} q(t) \phi_{+\varepsilon, \lambda}(t) d t .
\end{aligned}
$$

Proof For proving it is enough to substitute $s^{2} \phi_{-\varepsilon, \lambda}(t)+\phi_{-\varepsilon, \lambda}^{\prime \prime}(t), s^{2} \phi_{\varepsilon, \lambda}(t)+\phi_{\varepsilon, \lambda}^{\prime \prime}(t)$ and $s^{2} \phi_{+\varepsilon, \lambda}(t)+\phi_{+\varepsilon, \lambda}^{\prime \prime}(t)$ instead of $q(t) \phi_{-\varepsilon, \lambda}(t), q(t) \phi_{\varepsilon, \lambda}(t)$ and $q(t) \phi_{+\varepsilon, \lambda}(t)$ in the integral terms of (29), (30) and (31), respectively, and integrate by parts twice.

Lemma 3 Let $\lambda=s^{2}$. $\operatorname{Im} s=\ell$. Then the function $\phi_{\lambda}(x)$ has the following asymptotic representations for $|\lambda| \rightarrow \infty$, which hold uniformly for $x \in I_{i}(i=1,2,3)$ :

$$
\begin{aligned}
\phi_{-\varepsilon, \lambda}^{(k)}(x)= & \beta_{2}(\cos (s(x-a)))^{(k)}+O\left(|s|^{k-1} e^{|\ell|(x-a)}\right), \\
\phi_{\varepsilon, \lambda}^{(k)}(x)= & -s \mu_{2} \beta_{2} \sin \left(s\left(\theta_{-\varepsilon}-a\right)\right)\left(\cos \left(s\left(x-\theta_{-\varepsilon}\right)\right)\right)^{(k)}+O\left(|s|^{k} e^{|\ell|(x-a)}\right), \\
\phi_{+\varepsilon, \lambda}^{(k)}(x)= & s^{2} \mu_{2} \eta_{2} \beta_{2} \sin \left(s\left(\theta_{-\varepsilon}-a\right)\right) \sin \left(s\left(\theta_{+\varepsilon}-\theta_{-\varepsilon}\right)\right)\left(\cos \left(s\left(x-\theta_{+\varepsilon}\right)\right)\right)^{(k)} \\
& +O\left(|s|^{k+1} e^{|\ell|(x-a)}\right),
\end{aligned}
$$

for $\beta_{2} \neq 0$,

$$
\begin{aligned}
\phi_{-\varepsilon, \lambda}^{(k)}(x)= & -\frac{\beta_{1}}{s}(\sin (s(x-a)))^{(k)}+O\left(|s|^{k-2} e^{|\ell|(x-a)}\right), \\
\phi_{\varepsilon, \lambda}^{(k)}(x)= & -\mu_{2} \beta_{1} \cos \left(s\left(\theta_{-\varepsilon}-a\right)\right)\left(\cos \left(s\left(x-\theta_{-\varepsilon}\right)\right)\right)^{(k)}+O\left(|s|^{k-1} e^{|\ell|(x-a)}\right), \\
\phi_{+\varepsilon, \lambda}^{(k)}(x)= & s \mu_{2} \eta_{2} \beta_{1} \cos \left(s\left(\theta_{-\varepsilon}-a\right)\right) \sin \left(s\left(\theta_{+\varepsilon}-\theta_{-\varepsilon}\right)\right)\left(\cos \left(s\left(x-\theta_{+\varepsilon}\right)\right)\right)^{(k)} \\
& +O\left(|s|^{k} e^{|\ell|(x-a)}\right),
\end{aligned}
$$

for $\beta_{2}=0$. 
Proof These formulae can be proven similar to Titchmarsh's proof [20] and also the techniques in $[13,15]$.

Lemma 4 Let $\lambda=s^{2} . \operatorname{Im} s=\ell$. Then the characteristic function $\omega(\lambda)$ has the following asymptotic representations:

Case 1. If $\beta_{2} \neq 0, \alpha_{2}^{\prime} \neq 0$, then

$$
\begin{aligned}
\omega(\lambda)= & \frac{1}{D_{1} D_{2}} s^{5} \beta_{2} \alpha_{2}^{\prime} \mu_{2} \eta_{2} \sin \left(s\left(\theta_{-\varepsilon}-a\right)\right) \sin \left(s\left(\theta_{+\varepsilon}-\theta_{-\varepsilon}\right)\right) \sin \left(s\left(b-\theta_{+\varepsilon}\right)\right) \\
& +O\left(|s|^{4} e^{|\ell|(b-a)}\right) .
\end{aligned}
$$

Case 2. If $\beta_{2} \neq 0, \alpha_{2}^{\prime}=0$, then

$$
\begin{aligned}
\omega(\lambda)= & \frac{1}{D_{1} D_{2}} s^{4} \beta_{2} \alpha_{1}^{\prime} \mu_{2} \eta_{2} \sin \left(s\left(\theta_{-\varepsilon}-a\right)\right) \sin \left(s\left(\theta_{+\varepsilon}-\theta_{-\varepsilon}\right)\right) \cos \left(s\left(b-\theta_{+\varepsilon}\right)\right) \\
& +O\left(|s|^{3} e^{|\ell|(b-a)}\right) .
\end{aligned}
$$

Case 3. If $\beta_{2}=0, \alpha_{2}^{\prime} \neq 0$, then

$$
\begin{aligned}
\omega(\lambda)= & \frac{1}{D_{1} D_{2}} s^{4} \beta_{1} \alpha_{2}^{\prime} \mu_{2} \eta_{2} \cos \left(s\left(\theta_{-\varepsilon}-a\right)\right) \sin \left(s\left(\theta_{+\varepsilon}-\theta_{-\varepsilon}\right)\right) \sin \left(s\left(b-\theta_{+\varepsilon}\right)\right) \\
& +O\left(|s|^{3} e^{|\ell|(b-a)}\right) .
\end{aligned}
$$

Case 4. If $\beta_{2}=0, \alpha_{2}^{\prime}=0$, then

$$
\begin{aligned}
\omega(\lambda)= & \frac{1}{D_{1} D_{2}} s^{3} \beta_{1} \alpha_{1}^{\prime} \mu_{2} \eta_{2} \cos \left(s\left(\theta_{-\varepsilon}-a\right)\right) \sin \left(s\left(\theta_{+\varepsilon}-\theta_{-\varepsilon}\right)\right) \cos \left(s\left(b-\theta_{+\varepsilon}\right)\right) \\
& +O\left(|s|^{2} e^{|\ell|(b-a)}\right) .
\end{aligned}
$$

Proof The proof is immediate by substituting (34) and (37) into the representation

$$
\omega(\lambda)=\frac{1}{D_{1} D_{2}}\left\{\left(\lambda \alpha_{1}^{\prime}+\alpha_{1}\right) \phi_{+\varepsilon, \lambda}(b)-\left(\lambda \alpha_{2}^{\prime}+\alpha_{2}\right) \phi_{+\varepsilon, \lambda}^{\prime}(b)\right\} .
$$

Corollary 3 The eigenvalues of the problem (1)-(7) is bounded from below.

We are now ready to find the asymptotic approximation formulae for the eigenvalues of the problem (1)-(7). Since the eigenvalues coincide with the zeros of the entire functions $\omega(\lambda)$, it follows that they have no finite accumulation point. Moreover, all eigenvalues are real and bounded below by Corollaries 1 and 3 . Therefore, we may renumber them as $\lambda_{0} \leq$ $\lambda_{1} \leq \lambda_{2} \leq \cdots$, which are counted according to their multiplicity. Below we shall denote $s_{n}^{2}=\lambda_{n}$.

Theorem 2 The problem (1)-(7) has a precisely denumerable number of real eigenvalues, whose behaviour may be expressed by the three sequences $\left\{\lambda_{n}^{\prime}\right\},\left\{\lambda_{n}^{\prime \prime}\right\}$ and $\left\{\lambda_{n}^{\prime \prime \prime}\right\}$ with the following asymptotics representations for $n \rightarrow \infty$ : 
Case 1. If $\beta_{2} \neq 0, \alpha_{2}^{\prime} \neq 0$, then

$$
\begin{aligned}
& s_{n}^{\prime}=\frac{(n-1) \pi}{\left(\theta_{-\varepsilon}-a\right)}+O\left(\frac{1}{n}\right), \quad s_{n}^{\prime \prime}=\frac{(n-1) \pi}{\left(\theta_{+\varepsilon}-\theta_{-\varepsilon}\right)}+O\left(\frac{1}{n}\right), \\
& s_{n}^{\prime \prime \prime}=\frac{(n-2) \pi}{\left(b-\theta_{+\varepsilon}\right)}+O\left(\frac{1}{n}\right) .
\end{aligned}
$$

Case 2. If $\beta_{2} \neq 0, \alpha_{2}^{\prime}=0$, then

$$
\begin{aligned}
& s_{n}^{\prime}=\frac{(n-1) \pi}{\left(\theta_{-\varepsilon}-a\right)}+O\left(\frac{1}{n}\right), \quad s_{n}^{\prime \prime}=\frac{(n-1) \pi}{\left(\theta_{+\varepsilon}-\theta_{-\varepsilon}\right)}+O\left(\frac{1}{n}\right), \\
& s_{n}^{\prime \prime \prime}=\frac{(n-1 / 2) \pi}{\left(b-\theta_{+\varepsilon}\right)}+O\left(\frac{1}{n}\right) .
\end{aligned}
$$

Case 3. If $\beta_{2}=0, \alpha_{2}^{\prime} \neq 0$, then

$$
\begin{aligned}
& s_{n}^{\prime}=\frac{(n-1 / 2) \pi}{\left(\theta_{-\varepsilon}-a\right)}+O\left(\frac{1}{n}\right), \quad s_{n}^{\prime \prime}=\frac{(n-1) \pi}{\left(\theta_{+\varepsilon}-\theta_{-\varepsilon}\right)}+O\left(\frac{1}{n}\right), \\
& s_{n}^{\prime \prime \prime}=\frac{(n-1) \pi}{\left(b-\theta_{+\varepsilon}\right)}+O\left(\frac{1}{n}\right) .
\end{aligned}
$$

Case 4. If $\beta_{2}=0, \alpha_{2}^{\prime}=0$, then

$$
\begin{aligned}
& s_{n}^{\prime}=\frac{(n-1 / 2) \pi}{\left(\theta_{-\varepsilon}-a\right)}+O\left(\frac{1}{n}\right), \quad s_{n}^{\prime \prime}=\frac{(n-1) \pi}{\left(\theta_{+\varepsilon}-\theta_{-\varepsilon}\right)}+O\left(\frac{1}{n}\right), \\
& s_{n}^{\prime \prime \prime}=\frac{(n-1 / 2) \pi}{\left(b-\theta_{+\varepsilon}\right)}+O\left(\frac{1}{n}\right) .
\end{aligned}
$$

Proof We will only consider the first case. By applying the well-known Rouche theorem on a sufficiently large contour, it follows that $\omega(\lambda)$ has the same number of zeros inside the contour as the leading term in (38). Hence, if $\lambda_{0}^{\prime} \leq \lambda_{1}^{\prime} \leq \lambda_{2}^{\prime} \leq \cdots$ are the zeros of $\omega(\lambda)$ and $s_{n}^{2^{\prime}}=\lambda_{n}^{\prime}$ we have

$$
s_{n}^{\prime}=\frac{(n-1) \pi}{\left(\theta_{-\varepsilon}-a\right)}+\delta_{n}^{\prime}
$$

for sufficiently large $n$, where $\left|\delta_{n}^{\prime}\right| \leq \frac{\pi}{2\left(\theta_{-\varepsilon}-a\right)}$. By using (38) we have $\delta_{n}^{\prime}=O\left(\frac{1}{n}\right)$, which completes the proof for the first formula of Case 1 . The proof for the other cases are similar.

Then from (32)-(37) (for $k=0$ ) and the above theorem, the asymptotic behaviour of the eigenfunctions of the problem (1)-(7) is given by:

Case 1. If $\beta_{2} \neq 0, \alpha_{2}^{\prime} \neq 0$, then

$$
\phi_{\lambda_{n}^{\prime}}(x)= \begin{cases}\beta_{2} \cos \left(\frac{(n-1) \pi}{\left(\theta_{-\varepsilon}-a\right)}(x-a)\right)+O\left(\frac{1}{n}\right), & x \in\left[a, \theta_{-\varepsilon}\right), \\ O\left(\frac{1}{n}\right), & x \in\left(\theta_{-\varepsilon}, \theta_{+\varepsilon}\right), \\ O\left(\frac{1}{n}\right), & x \in\left(\theta_{+\varepsilon}, b\right],\end{cases}
$$




$$
\begin{gathered}
\phi_{\lambda_{n}^{\prime \prime}}(x)= \begin{cases}\beta_{2} \cos \left(\frac{(n-1) \pi}{\left(\theta_{+\varepsilon}-\theta_{-\varepsilon}\right)}(x-a)\right)+O\left(\frac{1}{n}\right), & x \in\left[a, \theta_{-\varepsilon}\right), \\
-\frac{(n-1) \pi}{\left(\theta_{+\varepsilon}-\theta_{-\varepsilon}\right)} \mu_{2} \beta_{2} \sin \left(\frac{(n-1) \pi}{\left(\theta_{+\varepsilon}-\theta_{-\varepsilon}\right)}\left(\theta_{-\varepsilon}-a\right)\right) \cos \left(\frac{(n-1) \pi}{\left(\theta_{+\varepsilon}-\theta_{-\varepsilon}\right)}\left(x-\theta_{-\varepsilon}\right)\right) & x \in\left(\theta_{-\varepsilon}, \theta_{+\varepsilon}\right), \\
\quad+O(1), & x \in\left(\theta_{+\varepsilon}, b\right], \\
O\left(\frac{1}{n}\right), & x \in\left[a, \theta_{-\varepsilon}\right),\end{cases} \\
\phi_{\lambda_{n}^{\prime \prime \prime}}(x)= \begin{cases}\beta_{2} \cos \left(\frac{(n-2) \pi}{\left(b-\theta_{+\varepsilon}\right)}(x-a)\right)+O\left(\frac{1}{n}\right), & x \in\left(\theta_{-\varepsilon}, \theta_{+\varepsilon}\right), \\
-\frac{(n-2) \pi}{\left(b-\theta_{+\varepsilon}\right)} \mu_{2} \beta_{2} \sin \left(\frac{(n-2) \pi}{\left(b-\theta_{+\varepsilon}\right)}\left(\theta_{-\varepsilon}-a\right)\right) \cos \left(\frac{(n-2) \pi}{\left(b-\theta_{+\varepsilon}\right)}\left(x-\theta_{-\varepsilon}\right)\right) & \\
\quad+O(1), & x \in\left(\theta_{+\varepsilon}, b\right] .\end{cases}
\end{gathered}
$$

Case 2. If $\beta_{2} \neq 0, \alpha_{2}^{\prime}=0$, then

$$
\begin{aligned}
& \phi_{\lambda_{n}^{\prime}}(x)= \begin{cases}\beta_{2} \cos \left(\frac{(n-1) \pi}{\left(\theta_{-\varepsilon}-a\right)}(x-a)\right)+O\left(\frac{1}{n}\right), & x \in\left[a, \theta_{-\varepsilon}\right), \\
O\left(\frac{1}{n}\right), & x \in\left(\theta_{-\varepsilon}, \theta_{+\varepsilon}\right), \\
O\left(\frac{1}{n}\right), & x \in\left(\theta_{+\varepsilon}, b\right],\end{cases} \\
& \phi_{\lambda_{n}^{\prime \prime}}(x)= \begin{cases}\beta_{2} \cos \left(\frac{(n-1) \pi}{\left(\theta_{+\varepsilon}-\theta_{-\varepsilon}\right)}(x-a)\right)+O\left(\frac{1}{n}\right), & x \in\left[a, \theta_{-\varepsilon}\right), \\
-\frac{(n-1) \pi}{\left(\theta_{+\varepsilon}-\theta_{-\varepsilon}\right)} \mu_{2} \beta_{2} \sin \left(\frac{(n-1) \pi}{\left(\theta_{+\varepsilon}-\theta_{-\varepsilon}\right)}\left(\theta_{-\varepsilon}-a\right)\right) \cos \left(\frac{(n-1) \pi}{\left(\theta_{+\varepsilon}-\theta_{-\varepsilon}\right)}\left(x-\theta_{-\varepsilon}\right)\right) & x \in\left(\theta_{-\varepsilon}, \theta_{+\varepsilon}\right), \\
+O(1), & x \in\left(\theta_{+\varepsilon}, b\right], \\
O\left(\frac{1}{n}\right), & x \in\left[a, \theta_{-\varepsilon}\right),\end{cases} \\
& \phi_{\lambda_{n}^{\prime \prime \prime}}(x)= \begin{cases}\beta_{2} \cos \left(\frac{(n-1 / 2) \pi}{\left(b-\theta_{+\varepsilon}\right)}(x-a)\right)+O\left(\frac{1}{n}\right), & x \in\left(\theta_{-\varepsilon}, \theta_{+\varepsilon}\right), \\
-\frac{(n-1 / 2) \pi}{\left(b-\theta_{+\varepsilon}\right)} \mu_{2} \beta_{2} \sin \left(\frac{(n-1 / 2) \pi}{\left(b-\theta_{+\varepsilon}\right)}\left(\theta_{-\varepsilon}-a\right)\right) \cos \left(\frac{(n-1 / 2) \pi}{\left(b-\theta_{+\varepsilon}\right)}\left(x-\theta_{-\varepsilon}\right)\right) & x \in\left(\theta_{+\varepsilon}, b\right] . \\
+O(1), & (n-1 / 2) \pi \\
\left(\frac{\left.n-\theta_{+\varepsilon}\right)}{2} \mu_{2} \eta_{2} \beta_{2} \sin \left(\frac{(n-1 / 2) \pi}{\left(b-\theta_{+\varepsilon}\right)}\left(\theta_{-\varepsilon}-a\right)\right) \sin \left(\frac{(n-1 / 2) \pi}{\left(b-\theta_{+\varepsilon}\right)}\left(\theta_{+\varepsilon}-\theta_{-\varepsilon}\right)\right)\right.\end{cases}
\end{aligned}
$$

Case 3. If $\beta_{2}=0, \alpha_{2}^{\prime} \neq 0$, then

$$
\begin{aligned}
& \phi_{\lambda_{n}^{\prime}}(x)= \begin{cases}-\frac{\beta_{1}\left(\theta_{-\varepsilon}-a\right)}{(n-1 / 2) \pi} \sin \left(\frac{(n-1 / 2) \pi}{\left(\theta_{-\varepsilon}-a\right)}(x-a)\right)+O\left(\frac{1}{n^{2}}\right), & x \in\left[a, \theta_{-\varepsilon}\right), \\
O\left(\frac{1}{n}\right), & x \in\left(\theta_{-\varepsilon}, \theta_{+\varepsilon}\right), \\
O(1), & x \in\left(b, \theta_{+\varepsilon}\right],\end{cases} \\
& \phi_{\lambda_{n}^{\prime \prime}}(x)= \begin{cases}-\frac{\beta_{1}\left(\theta_{+\varepsilon}-\theta_{-\varepsilon}\right)}{(n-1) \pi} \sin \left(\frac{(n-1) \pi}{\left(\theta_{+\varepsilon}-\theta_{-\varepsilon}\right)}(x-a)\right)+O\left(\frac{1}{n^{2}}\right), & x \in\left[a, \theta_{-\varepsilon}\right), \\
-\mu_{2} \beta_{1} \cos \left(\frac{(n-1) \pi}{\left(\theta_{+\varepsilon}-\theta_{-\varepsilon}\right)}\left(\theta_{-\varepsilon}-a\right)\right) \cos \left(\frac{(n-1) \pi}{\left(\theta_{+\varepsilon}-\theta_{-\varepsilon}\right)}\left(x-\theta_{-\varepsilon}\right)\right) & x \in\left(\theta_{-\varepsilon}, \theta_{+\varepsilon}\right), \\
+O\left(\frac{1}{n}\right), & x \in\left(b, \theta_{+\varepsilon}\right], \\
O(1), & \end{cases}
\end{aligned}
$$




$$
\phi_{\lambda_{n}^{\prime \prime \prime}}(x)= \begin{cases}-\frac{\beta_{1}\left(b-\theta_{+\varepsilon}\right)}{(n-1) \pi} \sin \left(\frac{(n-1) \pi}{\left(b-\theta_{+\varepsilon}\right)}(x-a)\right)+O\left(\frac{1}{n^{2}}\right), & x \in\left[a, \theta_{-\varepsilon}\right), \\ -\mu_{2} \beta_{1} \cos \left(\frac{(n-1) \pi}{\left(b-\theta_{+\varepsilon}\right)}\left(\theta_{-\varepsilon}-a\right)\right) \cos \left(\frac{(n-1) \pi}{\left(b-\theta_{+\varepsilon}\right)}\left(x-\theta_{-\varepsilon}\right)\right) & \\ \quad+O\left(\frac{1}{n}\right), & x \in\left(\theta_{-\varepsilon}, \theta_{+\varepsilon}\right), \\ \frac{(n-1) \pi}{\left(b-\theta_{+\varepsilon}\right)} \mu_{2} \beta_{1} \eta_{2} \cos \left(\frac{(n-1) \pi}{\left(b-\theta_{+\varepsilon}\right)}\left(\theta_{-\varepsilon}-a\right)\right) \sin \left(\frac{(n-1) \pi}{\left(b-\theta_{+\varepsilon}\right)}\left(\theta_{+\varepsilon}-\theta_{-\varepsilon}\right)\right) & \\ \quad \times \cos \left(\frac{(n-1) \pi}{\left(b-\theta_{+\varepsilon}\right)}\left(x-\theta_{-\varepsilon}\right)\right)+O(1), & x \in\left(b, \theta_{+\varepsilon}\right] .\end{cases}
$$

Case 4. If $\beta_{2}=0, \alpha_{2}^{\prime}=0$, then

$$
\begin{aligned}
& \phi_{\lambda_{n}^{\prime}}(x)= \begin{cases}-\frac{\beta_{1}\left(\theta_{-\varepsilon}-a\right)}{(n-1 / 2) \pi} \sin \left(\frac{(n-1 / 2) \pi}{\left(\theta_{-\varepsilon}-a\right)}(x-a)\right)+O\left(\frac{1}{n^{2}}\right), & x \in\left[a, \theta_{-\varepsilon}\right), \\
O\left(\frac{1}{n}\right), & x \in\left(\theta_{-\varepsilon}, \theta_{+\varepsilon}\right), \\
O(1), & x \in\left(b, \theta_{+\varepsilon}\right],\end{cases} \\
& \phi_{\lambda_{n}^{\prime \prime}}(x)= \begin{cases}-\frac{\beta_{1}\left(\theta_{+\varepsilon}-\theta_{-\varepsilon}\right)}{(n-1) \pi} \sin \left(\frac{(n-1) \pi}{\left(\theta_{+\varepsilon}-\theta_{-\varepsilon}\right)}(x-a)\right)+O\left(\frac{1}{n^{2}}\right), & x \in\left[a, \theta_{-\varepsilon}\right), \\
-\mu_{2} \beta_{1} \cos \left(\frac{(n-1) \pi}{\left(\theta_{+\varepsilon}-\theta_{-\varepsilon}\right)}\left(\theta_{-\varepsilon}-a\right)\right) \cos \left(\frac{(n-1) \pi}{\left(\theta_{+\varepsilon}-\theta_{-\varepsilon}\right)}\left(x-\theta_{-\varepsilon}\right)\right) & x \in\left(\theta_{-\varepsilon}, \theta_{+\varepsilon}\right), \\
+O\left(\frac{1}{n}\right), & x \in\left(b, \theta_{+\varepsilon}\right], \\
O(1), & x \in\left[a, \theta_{-\varepsilon}\right),\end{cases} \\
& \phi_{\lambda_{n}^{\prime \prime}}(x)= \begin{cases}-\frac{\beta_{1}\left(b-\theta_{+\varepsilon}\right)}{(n-1 / 2) \pi} \sin \left(\frac{(n-1 / 2) \pi}{\left(b-\theta_{+\varepsilon}\right)}(x-a)\right)+O\left(\frac{1}{n^{2}}\right), & x \in\left(\theta_{-\varepsilon}, \theta_{+\varepsilon}\right), \\
-\mu_{2} \beta_{1} \cos \left(\frac{(n-1 / 2) \pi}{\left(b-\theta_{+\varepsilon}\right)}\left(\theta_{-\varepsilon}-a\right)\right) \cos \left(\frac{(n-1 / 2) \pi}{\left(b-\theta_{+\varepsilon}\right)}\left(x-\theta_{-\varepsilon}\right)\right) & \\
+O\left(\frac{1}{n}\right), & x \in\left(b, \theta_{+\varepsilon}\right] . \\
\frac{(n-1 / 2) \pi}{\left(b-\theta_{+\varepsilon}\right)} \mu_{2} \beta_{1} \eta_{2} \cos \left(\frac{(n-1 / 2) \pi}{\left(b-\theta_{+\varepsilon}\right)}\left(\theta_{-\varepsilon}-a\right)\right) \sin \left(\frac{(n-1 / 2) \pi}{\left(b-\theta_{+\varepsilon}\right)}\left(\theta_{+\varepsilon}-\theta_{-\varepsilon}\right)\right) & \\
\quad \times \cos \left(\frac{(n-1 / 2) \pi}{\left(b-\theta_{+\varepsilon}\right)}\left(x-\theta_{-\varepsilon}\right)\right)+O(1), & \end{cases}
\end{aligned}
$$

All these asymptotic formulae hold uniformly for $x \in I$.

\section{Green's function}

Let $F=\left(\begin{array}{c}f(x) \\ h\end{array}\right)$ be a continuous function. To study the completeness of the eigenelements of $A$, and hence the completeness of the eigenfunctions (1)-(7), we derive Green's function of the problem (1)-(7) as well as the resolvent of $A$. Indeed let $\lambda \in \mathbb{C}$ not be an eigenvalue of $A$ and consider the inhomogeneous problem

$$
(\lambda \mathrm{I}-A) Y=F, \quad x \in I
$$

where I is the identity operator and $Y=\left(\begin{array}{c}y(x) \\ R^{\prime}(y)\end{array}\right) \in D(A)$. Since

$$
(\lambda \mathrm{I}-A) Y=\lambda\left(\begin{array}{c}
y(x) \\
R^{\prime}(y)
\end{array}\right)-\left(\begin{array}{c}
\tau(y) \\
-R(y)
\end{array}\right)=\left(\begin{array}{c}
f(x) \\
h
\end{array}\right),
$$

we have

$$
\begin{aligned}
& (\lambda-\tau) y(x)=f(x), \quad x \in I, \\
& \lambda R^{\prime}(y)+R(y)=h .
\end{aligned}
$$


Now we can represent the general solution of homogeneous differential equation (1), appropriate to (49) in the following form:

$$
y(x, \lambda)= \begin{cases}c_{1} \phi_{-\varepsilon, \lambda}(x)+c_{2} \chi_{-\varepsilon, \lambda}(x), & x \in\left[a, \theta_{-\varepsilon}\right) \\ c_{3} \phi_{\varepsilon, \lambda}(x)+c_{4} \chi_{\varepsilon, \lambda}(x), & x \in\left(\theta_{-\varepsilon}, \theta_{+\varepsilon}\right) \\ c_{5} \phi_{+\varepsilon, \lambda}(x)+c_{6} \chi_{+\varepsilon, \lambda}(x), & x \in\left(\theta_{+\varepsilon}, b\right]\end{cases}
$$

in which $c_{i}(i=\overline{1,6})$ are arbitrary constants. By applying the method of variation of the constants, we shall search the general solution of the non-homogeneous linear differential equation (49) in the following form:

$$
y(x, \lambda)= \begin{cases}c_{1}(x, \lambda) \phi_{-\varepsilon, \lambda}(x)+c_{2}(x, \lambda) \chi_{-\varepsilon, \lambda}(x), & x \in\left[a, \theta_{-\varepsilon}\right), \\ c_{3}(x, \lambda) \phi_{\varepsilon, \lambda}(x)+c_{4}(x, \lambda) \chi_{\varepsilon, \lambda}(x), & x \in\left(\theta_{-\varepsilon}, \theta_{+\varepsilon}\right), \\ c_{5}(x, \lambda) \phi_{+\varepsilon, \lambda}(x)+c_{6}(x, \lambda) \chi_{+\varepsilon, \lambda}(x), & x \in\left(\theta_{+\varepsilon}, b\right]\end{cases}
$$

where the functions $c_{i}(x, \lambda)(i=\overline{1,6})$ satisfy the linear system of equations

$$
\begin{aligned}
& \left\{\begin{array}{l}
c_{1}^{\prime}(x, \lambda) \phi_{-\varepsilon, \lambda}(x)+c_{2}^{\prime}(x, \lambda) \chi_{-\varepsilon, \lambda}(x)=0, \\
c_{1}^{\prime}(x, \lambda) \phi_{-\varepsilon, \lambda}^{\prime}(x)+c_{2}^{\prime}(x, \lambda) \chi_{-\varepsilon, \lambda}^{\prime}(x)=f(x),
\end{array} \text { for } x \in\left[a, \theta_{-\varepsilon}\right),\right. \\
& \left\{\begin{array}{l}
c_{3}^{\prime}(x, \lambda) \phi_{\varepsilon, \lambda}(x)+c_{4}^{\prime}(x, \lambda) \chi_{\varepsilon, \lambda}(x)=0, \\
c_{3}^{\prime}(x, \lambda) \phi_{\varepsilon, \lambda}^{\prime}(x)+c_{4}^{\prime}(x, \lambda) \chi_{\varepsilon, \lambda}^{\prime}(x)=f(x),
\end{array} \text { for } x \in\left(\theta_{-\varepsilon}, \theta_{+\varepsilon}\right),\right. \\
& \left\{\begin{array}{l}
c_{5}^{\prime}(x, \lambda) \phi_{+\varepsilon, \lambda}(x)+c_{6}^{\prime}(x, \lambda) \chi_{+\varepsilon, \lambda}(x)=0, \\
c_{5}^{\prime}(x, \lambda) \phi_{+\varepsilon, \lambda}^{\prime}(x)+c_{6}^{\prime}(x, \lambda) \chi_{+\varepsilon, \lambda}^{\prime}(x)=f(x),
\end{array}\right.
\end{aligned}
$$

Since $\lambda$ is not an eigenvalue and $\omega_{-\varepsilon}(\lambda) \neq 0, \omega_{\varepsilon}(\lambda) \neq 0$ and $\omega_{+\varepsilon}(\lambda) \neq 0$, each of the linear systems in (52)-(54) has a unique solution, which leads to

$$
\begin{aligned}
& c_{1}(x, \lambda)=\frac{1}{\omega_{-\varepsilon}(\lambda)} \int_{x}^{\theta_{-\varepsilon}} \chi_{-\varepsilon, \lambda}(t) f(t) d t+c_{1}(\lambda), \\
& c_{2}(x, \lambda)=\frac{1}{\omega_{-\varepsilon}(\lambda)} \int_{a}^{x} \phi_{-\varepsilon, \lambda}(t) f(t) d t+c_{2}(\lambda), \\
& c_{3}(x, \lambda)=\frac{1}{\omega_{\varepsilon}(\lambda)} \int_{x}^{\theta_{+\varepsilon}} \chi_{\varepsilon, \lambda}(t) f(t) d t+c_{3}(\lambda), \\
& c_{4}(x, \lambda)=\frac{1}{\omega_{\varepsilon}(\lambda)} \int_{\theta_{-\varepsilon}}^{x} \phi_{\varepsilon, \lambda}(t) f(t) d t+c_{4}(\lambda), \\
& c_{5}(x, \lambda)=\frac{1}{\omega_{+\varepsilon}(\lambda)} \int_{x}^{b} \chi_{+\varepsilon, \lambda}(t) f(t) d t+c_{5}(\lambda), \\
& c_{6}(x, \lambda)=\frac{1}{\omega_{+\varepsilon}(\lambda)} \int_{\theta_{+\varepsilon}}^{x} \phi_{+\varepsilon, \lambda}(t) f(t) d t+c_{6}(\lambda),
\end{aligned}
$$


where $c_{i}(\lambda)(i=\overline{1,6})$ are arbitrary constants. Substituting (55) into (51), we obtain the solution of (49),

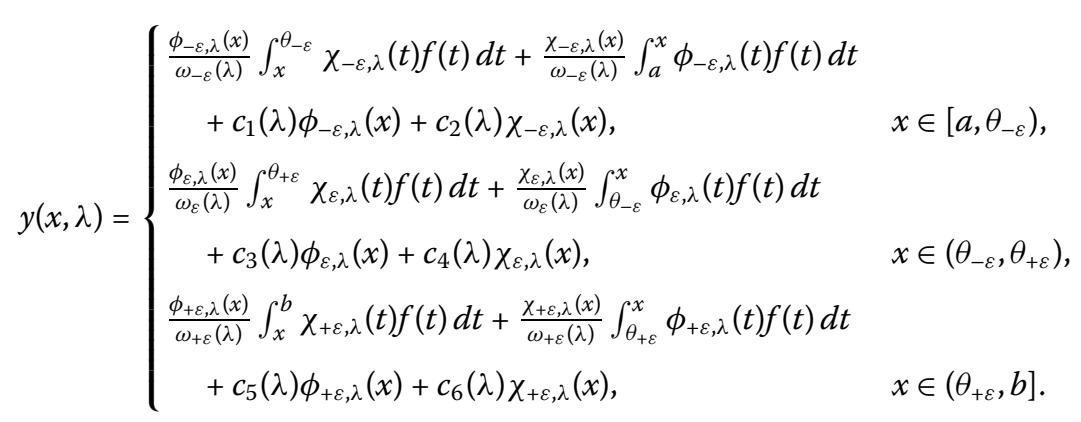

Then from (2), (50) and (4)-(7), we get

$$
\begin{aligned}
& c_{1}(\lambda)=\frac{1}{\omega_{\varepsilon}(\lambda)} \int_{\theta_{-\varepsilon}}^{\theta_{+\varepsilon}} \chi_{\varepsilon, \lambda}(t) f(t) d t+\frac{1}{\omega_{+\varepsilon}(\lambda)} \int_{\theta_{+\varepsilon}}^{b} \chi_{+\varepsilon, \lambda}(t) f(t) d t+\frac{h}{\omega_{+\varepsilon}(\lambda)}, \\
& c_{2}(\lambda)=0 \\
& c_{3}(\lambda)=\frac{1}{\omega_{+\varepsilon}(\lambda)} \int_{\theta_{+\varepsilon}}^{b} \chi_{+\varepsilon, \lambda}(t) f(t) d t+\frac{h}{\omega_{+\varepsilon}(\lambda)}, \\
& c_{4}(\lambda)=\frac{1}{\omega_{-\varepsilon}(\lambda)} \int_{a}^{\theta_{-\varepsilon}} \phi_{-\varepsilon, \lambda}(t) f(t) d t, \\
& c_{5}(\lambda)=\frac{h}{\omega_{+\varepsilon}(\lambda)}, \\
& c_{6}(\lambda)=\frac{1}{\omega_{-\varepsilon}(\lambda)} \int_{a}^{\theta_{-\varepsilon}} \phi_{-\varepsilon, \lambda}(t) f(t) d t+\frac{1}{\omega_{\varepsilon}(\lambda)} \int_{\theta_{-\varepsilon}}^{\theta_{+\varepsilon}} \phi_{\varepsilon, \lambda}(t) f(t) d t .
\end{aligned}
$$

Substituting (57) and (26) into (56), then (56) can be written as

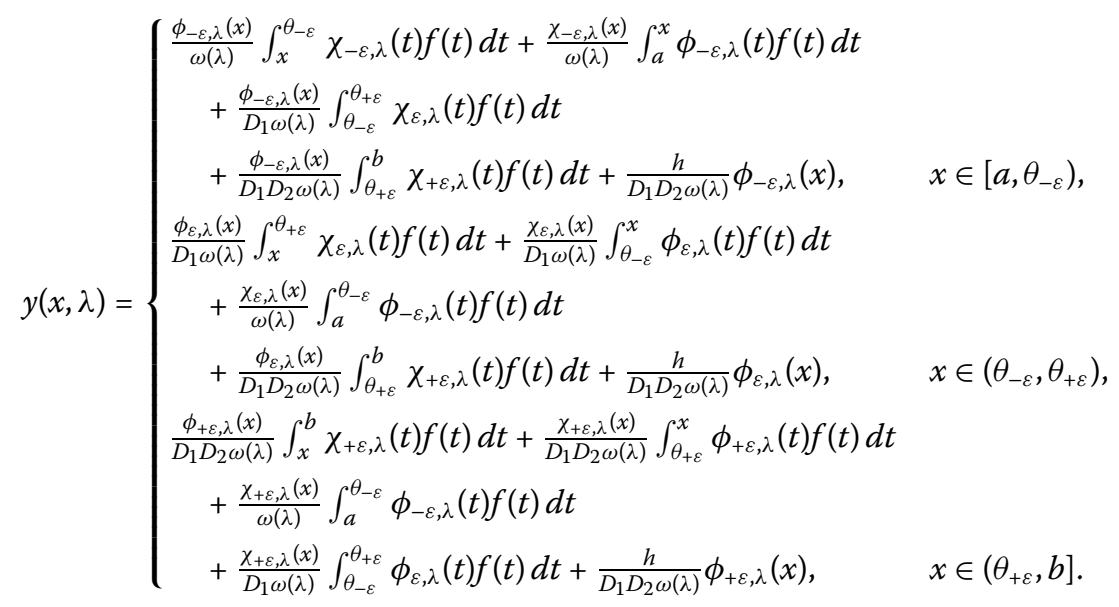

Then (58) can be rewritten in the form

$$
\begin{aligned}
y(x, \lambda)= & \int_{a}^{\theta_{-\varepsilon}} G(x, t ; \lambda) f(t) d t+\frac{1}{D_{1}} \int_{\theta_{-\varepsilon}}^{\theta_{+\varepsilon}} G(x, t ; \lambda) f(t) d t \\
& +\frac{1}{D_{1} D_{2}} \int_{\theta_{+\varepsilon}}^{b} G(x, t ; \lambda) f(t) d t+\frac{h \phi_{\lambda}(x)}{D_{1} D_{2} \omega(\lambda)},
\end{aligned}
$$


where

$$
G(x, t ; \lambda)= \begin{cases}\frac{\phi_{\lambda}(t) \chi_{\lambda}(x)}{\omega(\lambda)}, & a \leq t \leq x \leq b, x \neq \theta_{-\varepsilon}, \theta_{+\varepsilon} ; t \neq \theta_{-\varepsilon}, \theta_{+\varepsilon} \\ \frac{\phi_{\lambda}(x) \chi_{\lambda}(t)}{\omega(\lambda)}, & a \leq x \leq t \leq b, x \neq \theta_{-\varepsilon}, \theta_{+\varepsilon} ; \neq \neq \theta_{-\varepsilon}, \theta_{+\varepsilon}\end{cases}
$$

is the Green's function of the problem (1)-(7).

Hence, we have

$$
\begin{aligned}
Y & =(\lambda \mathrm{I}-A)^{-1} F \\
& =\left(\begin{array}{c}
\left.\int_{a}^{\theta-\varepsilon} G(x, t ; \lambda) f(t) d t+\frac{1}{D_{1}} \int_{\theta_{-\varepsilon}}^{\theta_{+\varepsilon}} G(x, t ; \lambda) f(t) d t+\frac{1}{D_{1} D_{2}} \int_{\theta_{+\varepsilon}}^{b} G(x, t ; \lambda) f(t) d t+\frac{h \phi_{\lambda}(x)}{D_{1} D_{2} \omega(\lambda)}\right) \\
R^{\prime}(y)
\end{array}\right.
\end{aligned}
$$

the resolvent operator of the problem (1)-(7).

\section{Example}

We indicate in this example the effect on determining the discontinuities of different values of $\theta$ and $\varepsilon$. For each value of $\theta$ and $\varepsilon$, we shall display the characteristic function and give the first five eigenvalues of the problem.

Example Consider the boundary value problem

$$
\begin{aligned}
& -y^{\prime \prime}=\lambda y, \\
& y^{\prime}(-2)=0, \\
& \lambda y(4)-y^{\prime}(4)=0, \\
& y\left(\theta_{-\varepsilon}+\right)=2 y\left(\theta_{-\varepsilon}\right), \\
& y^{\prime}\left(\theta_{-\varepsilon}+\right)=y\left(\theta_{-\varepsilon}-\right)+2^{-1} y^{\prime}\left(\theta_{-\varepsilon}-\right), \\
& y\left(\theta_{+\varepsilon}+\right)=2^{-1} y\left(\theta_{+\varepsilon}-\right), \\
& y^{\prime}\left(\theta_{+\varepsilon}+\right)=y\left(\theta_{+\varepsilon}-\right)+2 y^{\prime}\left(\theta_{+\varepsilon}-\right),
\end{aligned}
$$

where $I=[-2,4]$.

Let $\lambda=s^{2}$. The eigenvalues of the problem (61)-(67) are the squares of the zeros of the characteristic function of $\omega(\lambda)$, given by

$$
\begin{aligned}
\omega(\lambda)= & s^{2}\left\{\cos \left(s\left(\theta_{-\varepsilon}+2\right)\right) \cos \left(s\left(\theta_{+\varepsilon}-\theta_{-\varepsilon}\right)\right) \cos \left(s\left(4-\theta_{+\varepsilon}\right)\right)\right. \\
& -\frac{1}{4} \sin \left(s\left(\theta_{-\varepsilon}+2\right)\right) \sin \left(s\left(\theta_{+\varepsilon}-\theta_{-\varepsilon}\right)\right) \cos \left(s\left(4-\theta_{+\varepsilon}\right)\right) \\
& -4 \cos \left(s\left(\theta_{-\varepsilon}+2\right)\right) \sin \left(s\left(\theta_{+\varepsilon}-\theta_{-\varepsilon}\right)\right) \sin \left(s\left(4-\theta_{+\varepsilon}\right)\right) \\
& \left.-\sin \left(s\left(\theta_{-\varepsilon}+2\right)\right) \cos \left(s\left(\theta_{+\varepsilon}-\theta_{-\varepsilon}\right)\right) \sin \left(s\left(4-\theta_{+\varepsilon}\right)\right)\right\} \\
& +s\left\{5 \cos \left(s\left(\theta_{-\varepsilon}+2\right)\right) \cos \left(s\left(\theta_{+\varepsilon}-\theta_{-\varepsilon}\right)\right) \sin \left(s\left(4-\theta_{+\varepsilon}\right)\right)\right. \\
& -\frac{3}{4} \sin \left(s\left(\theta_{-\varepsilon}+2\right)\right) \sin \left(s\left(\theta_{+\varepsilon}-\theta_{-\varepsilon}\right)\right) \sin \left(s\left(4-\theta_{+\varepsilon}\right)\right)
\end{aligned}
$$




$$
\begin{aligned}
& +\frac{9}{2} \cos \left(s\left(\theta_{-\varepsilon}+2\right)\right) \sin \left(s\left(\theta_{+\varepsilon}-\theta_{-\varepsilon}\right)\right) \cos \left(s\left(4-\theta_{+\varepsilon}\right)\right) \\
& \left.+\sin \left(s\left(\theta_{-\varepsilon}+2\right)\right) \cos \left(s\left(\theta_{+\varepsilon}-\theta_{-\varepsilon}\right)\right) \cos \left(s\left(4-\theta_{+\varepsilon}\right)\right)\right\} \\
& +\frac{3}{2} \cos \left(s\left(\theta_{-\varepsilon}+2\right)\right) \sin \left(s\left(\theta_{+\varepsilon}-\theta_{-\varepsilon}\right)\right) \sin \left(s\left(4-\theta_{+\varepsilon}\right)\right) \\
& -4 \cos \left(s\left(\theta_{-\varepsilon}+2\right)\right) \cos \left(s\left(\theta_{+\varepsilon}-\theta_{-\varepsilon}\right)\right) \cos \left(s\left(4-\theta_{+\varepsilon}\right)\right) \\
& +\frac{1}{2} \sin \left(s\left(\theta_{-\varepsilon}+2\right)\right) \sin \left(s\left(\theta_{+\varepsilon}-\theta_{-\varepsilon}\right)\right) \cos \left(s\left(4-\theta_{+\varepsilon}\right)\right) \\
& -\frac{1}{s} \cos \left(s\left(\theta_{-\varepsilon}+2\right)\right) \sin \left(s\left(\theta_{+\varepsilon}-\theta_{-\varepsilon}\right)\right) \cos \left(s\left(4-\theta_{+\varepsilon}\right)\right) \\
& =0 .
\end{aligned}
$$

(1) Let $\theta=-1$ be an interior point in $I=[-2,4]$. From (8), we get $0<\varepsilon<1$.

(i) If $\varepsilon=1 / 4$, then the points of discontinuity are $\theta_{-\varepsilon}=-5 / 4$ and $\theta_{+\varepsilon}=-3 / 4$. Equation (68) is then reduced to

$$
\begin{aligned}
\omega(\lambda)= & s^{2}\left\{\cos \left(\frac{3}{4} s\right) \cos \left(\frac{1}{2} s\right) \cos \left(\frac{19}{4} s\right)-\frac{1}{4} \sin \left(\frac{3}{4} s\right) \sin \left(\frac{1}{2} s\right) \cos \left(\frac{19}{4} s\right)\right. \\
& \left.-4 \cos \left(\frac{3}{4} s\right) \sin \left(\frac{1}{2} s\right) \sin \left(\frac{19}{4} s\right)-\sin \left(\frac{3}{4} s\right) \cos \left(\frac{1}{2} s\right) \sin \left(\frac{19}{4} s\right)\right\} \\
& +s\left\{5 \cos \left(\frac{3}{4} s\right) \cos \left(\frac{1}{2} s\right) \sin \left(\frac{19}{4} s\right)-\frac{3}{4} \sin \left(\frac{3}{4} s\right) \sin \left(\frac{1}{2} s\right) \sin \left(\frac{19}{4} s\right)\right. \\
& \left.+\frac{9}{2} \cos \left(\frac{3}{4} s\right) \sin \left(\frac{1}{2} s\right) \cos \left(\frac{19}{4} s\right)+\sin \left(\frac{3}{4} s\right) \cos \left(\frac{1}{2} s\right) \cos \left(\frac{19}{4} s\right)\right\} \\
& +\frac{3}{2} \cos \left(\frac{3}{4} s\right) \sin \left(\frac{1}{2} s\right) \sin \left(\frac{19}{4} s\right)-4 \cos \left(\frac{3}{4} s\right) \cos \left(\frac{1}{2} s\right) \cos \left(\frac{19}{4} s\right) \\
& +\frac{1}{2} \sin \left(\frac{3}{4} s\right) \sin \left(\frac{1}{2} s\right) \cos \left(\frac{19}{4} s\right)-\frac{1}{s} \cos \left(\frac{3}{4} s\right) \sin \left(\frac{1}{2} s\right) \cos \left(\frac{19}{4} s\right) \\
= & 0 .
\end{aligned}
$$

The graph of the characteristic function for $\varepsilon=1 / 4$ is displayed in Figure 1 .

(ii) If $\varepsilon=1 / 2$, then the points of discontinuity are $\theta_{-\varepsilon}=-3 / 2$ and $\theta_{+\varepsilon}=-1 / 2$. Equation (68) is then reduced to

$$
\begin{aligned}
\omega(\lambda)= & s^{2}\left\{\cos \left(\frac{1}{2} s\right) \cos (s) \cos \left(\frac{9}{2} s\right)-\frac{1}{4} \sin \left(\frac{1}{2} s\right) \sin (s) \cos \left(\frac{9}{2} s\right)\right. \\
& \left.-4 \cos \left(\frac{1}{2} s\right) \sin (s) \sin \left(\frac{9}{2} s\right)-\sin \left(\frac{1}{2} s\right) \cos (s) \sin \left(\frac{9}{2} s\right)\right\} \\
& +s\left\{5 \cos \left(\frac{1}{2} s\right) \cos (s) \sin \left(\frac{9}{2} s\right)-\frac{3}{4} \sin \left(\frac{1}{2} s\right) \sin (s) \sin \left(\frac{9}{2} s\right)\right. \\
& \left.+\frac{9}{2} \cos \left(\frac{1}{2} s\right) \sin (s) \cos \left(\frac{9}{2} s\right)+\sin \left(\frac{1}{2} s\right) \cos (s) \cos \left(\frac{9}{2} s\right)\right\} \\
& +\frac{3}{2} \cos \left(\frac{1}{2} s\right) \sin (s) \sin \left(\frac{9}{2} s\right)-4 \cos \left(\frac{1}{2} s\right) \cos (s) \cos \left(\frac{9}{2} s\right)
\end{aligned}
$$




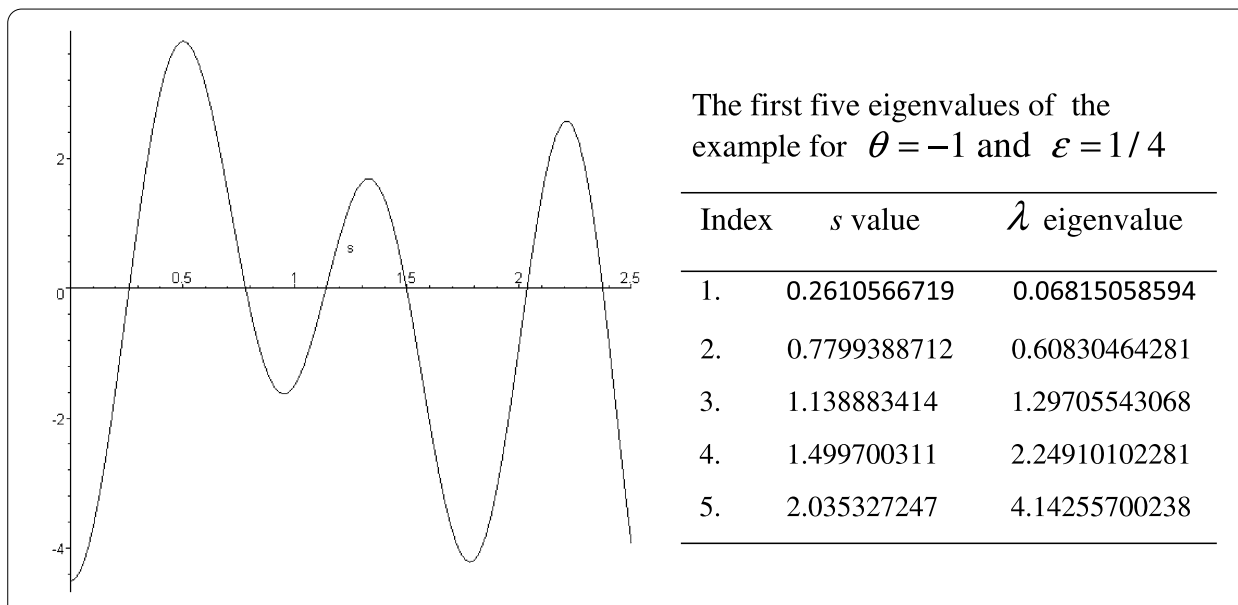

Figure 1 The graph of the characteristic function for $\theta=-1$ and $\varepsilon=1 / 4$.

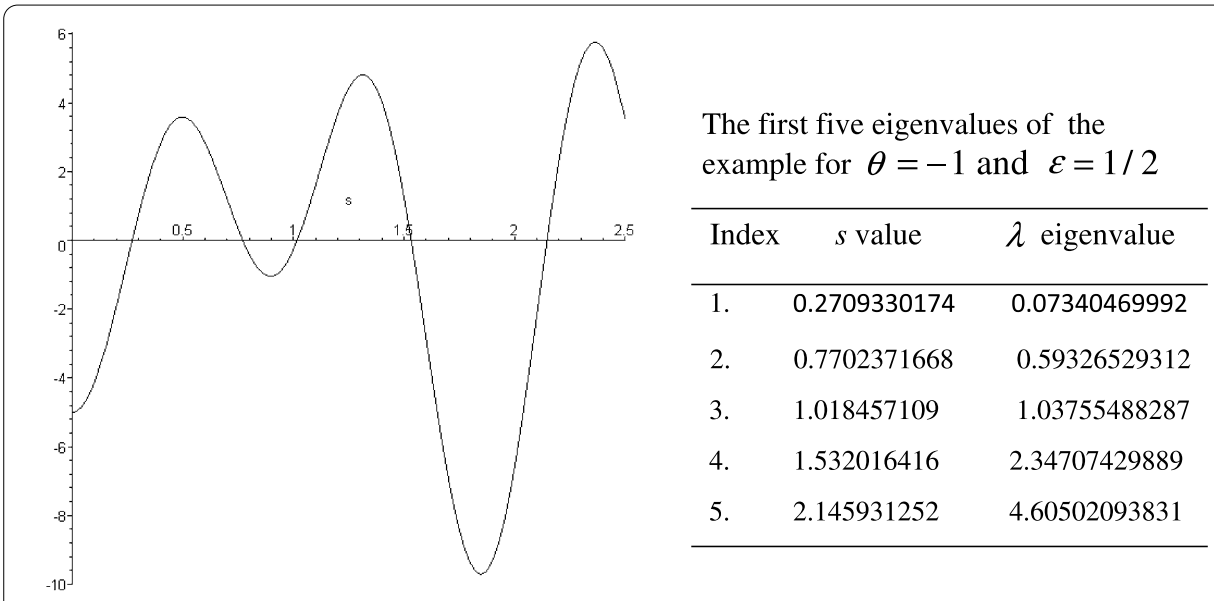

Figure 2 The graph of the characteristic function for $\theta=-1$ and $\varepsilon=1 / 2$.

$$
\begin{aligned}
& +\frac{1}{2} \sin \left(\frac{1}{2} s\right) \sin (s) \cos \left(\frac{9}{2} s\right)-\frac{1}{s} \cos \left(\frac{1}{2} s\right) \sin (s) \cos \left(\frac{9}{2} s\right) \\
= & 0 .
\end{aligned}
$$

The graph of the characteristic function for $\varepsilon=1 / 2$ is displayed in Figure 2 .

(2) Let $\theta=1$ be midpoint of the interval $I=[-2,4]$. From (8), we get $0<\varepsilon<3$.

(i) If $\varepsilon=1$, then the points of discontinuity are $\theta_{-\varepsilon}=0$ and $\theta_{+\varepsilon}=2$. Equation (68) is then reduced to

$$
\begin{aligned}
\omega(\lambda)= & s^{2}\left\{\cos ^{3}(2 s)-\frac{21}{4} \cos (2 s) \sin ^{2}(2 s)\right\} \\
& +s\left\{\frac{21}{2} \sin (2 s) \cos ^{2}(2 s)-\frac{3}{4} \sin ^{3}(2 s)\right\} \\
& +2 \cos (2 s) \sin ^{2}(2 s)-4 \cos ^{3}(2 s)-\frac{1}{s} \sin (2 s) \cos ^{2}(2 s) \\
= & 0 .
\end{aligned}
$$


The graph of the characteristic function for $\varepsilon=1$ is displayed in Figure 3.

(ii) If $\varepsilon=3 / 2$, then the points of discontinuity are $\theta_{-\varepsilon}=-1 / 2$ and $\theta_{+\varepsilon}=5 / 2$. The equation (68) is then reduced to

$$
\begin{aligned}
\omega(\lambda)= & s^{2}\left\{\cos ^{2}(3 s)-\frac{17}{8} \sin ^{2}(3 s)\right\} \\
& +s\left\{\frac{3}{2} \sin (6 s)-\frac{3}{4} \sin (3 s) \sin ^{2}\left(\frac{3}{2} s\right)+\frac{9}{2} \sin (3 s) \cos ^{2}\left(\frac{3}{2} s\right)\right\} \\
& +\sin ^{2}(3 s)-4 \cos (3 s) \cos ^{2}\left(\frac{3}{2} s\right)-\frac{1}{s} \sin (3 s) \cos ^{2}\left(\frac{3}{2} s\right) \\
= & 0 .
\end{aligned}
$$

The graph of the characteristic function for $\varepsilon=3 / 2$ is displayed in Figure 4 .

(3) Let $\theta=3$ be an interior point in $I=[-2,4]$. From (8), we get $0<\varepsilon<1$.
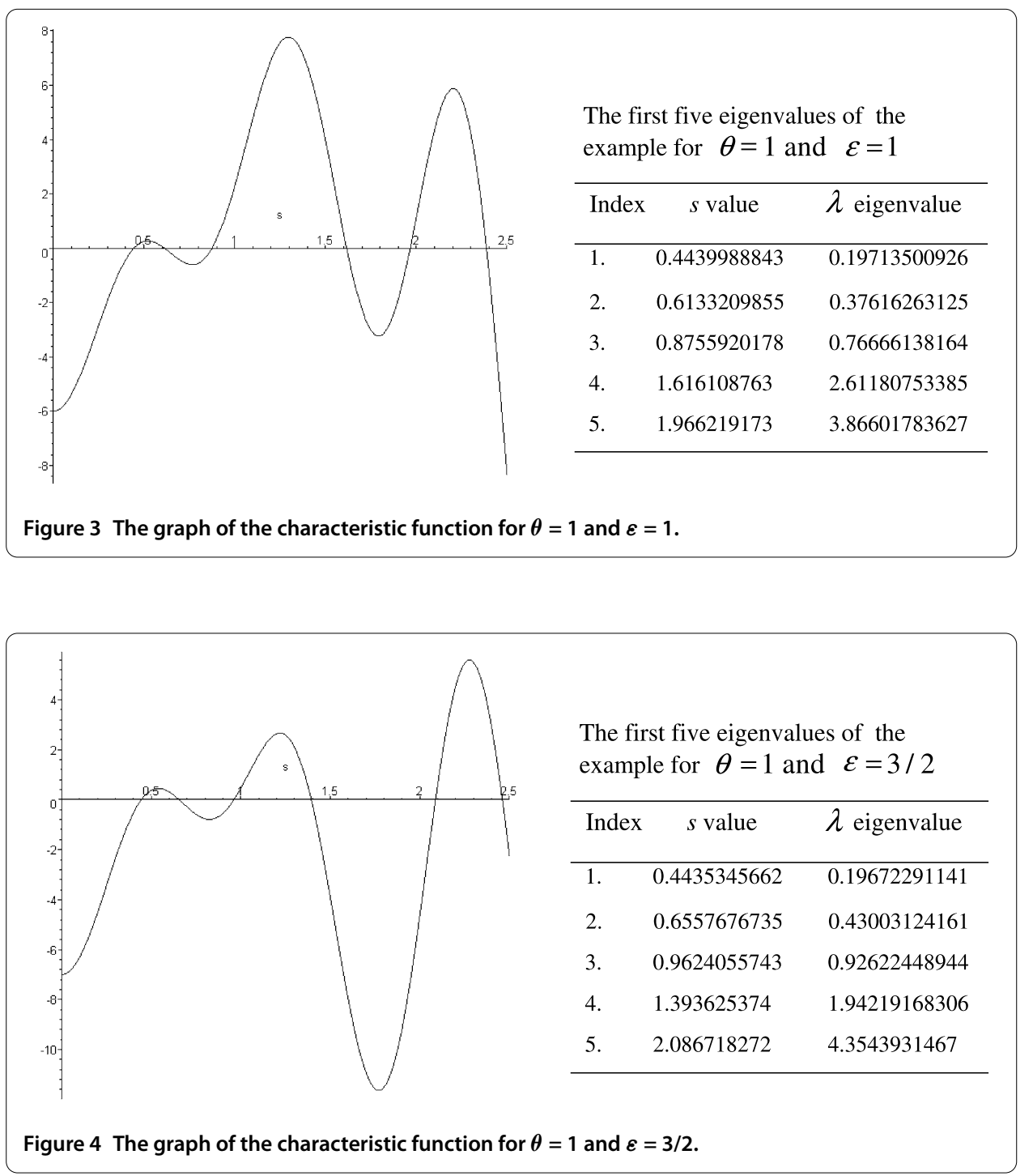
(i) If $\varepsilon=1 / 4$, then the points of discontinuity are $\theta_{-\varepsilon}=11 / 4$ and $\theta_{+\varepsilon}=13 / 4$. Equation (68) is then reduced to

$$
\begin{aligned}
\omega(\lambda)= & s^{2}\left\{\cos \left(\frac{19}{4} s\right) \cos \left(\frac{1}{2} s\right) \cos \left(\frac{3}{4} s\right)-\frac{1}{4} \sin \left(\frac{19}{4} s\right) \sin \left(\frac{1}{2} s\right) \cos \left(\frac{3}{4} s\right)\right. \\
& \left.-4 \cos \left(\frac{19}{4} s\right) \sin \left(\frac{1}{2} s\right) \sin \left(\frac{3}{4} s\right)-\sin \left(\frac{19}{4} s\right) \cos \left(\frac{1}{2} s\right) \sin \left(\frac{3}{4} s\right)\right\} \\
& +s\left\{5 \cos \left(\frac{19}{4} s\right) \cos \left(\frac{1}{2} s\right) \sin \left(\frac{3}{4} s\right)-\frac{3}{4} \sin \left(\frac{19}{4} s\right) \sin \left(\frac{1}{2} s\right) \sin \left(\frac{3}{4} s\right)\right. \\
& \left.+\frac{9}{2} \cos \left(\frac{19}{4} s\right) \sin \left(\frac{1}{2} s\right) \cos \left(\frac{3}{4} s\right)+\sin \left(\frac{19}{4} s\right) \cos \left(\frac{1}{2} s\right) \cos \left(\frac{3}{4} s\right)\right\} \\
& +\frac{3}{2} \cos \left(\frac{19}{4} s\right) \sin \left(\frac{1}{2} s\right) \sin \left(\frac{3}{4} s\right)-4 \cos \left(\frac{19}{4} s\right) \cos \left(\frac{1}{2} s\right) \cos \left(\frac{3}{4} s\right) \\
& +\frac{1}{2} \sin \left(\frac{19}{4} s\right) \sin \left(\frac{1}{2} s\right) \cos \left(\frac{3}{4} s\right)-\frac{1}{s} \cos \left(\frac{19}{4} s\right) \sin \left(\frac{1}{2} s\right) \cos \left(\frac{3}{4} s\right) \\
= & 0 .
\end{aligned}
$$

The graph of the characteristic function for $\varepsilon=1 / 4$ is displayed in Figure 5 .

(ii) If $\varepsilon=1 / 2$, then the points of discontinuity are $\theta_{-\varepsilon}=5 / 2$ and $\theta_{+\varepsilon}=7 / 2$. Equation (68) is then reduced to

$$
\begin{aligned}
\omega(\lambda)= & s^{2}\left\{\cos \left(\frac{9}{2} s\right) \cos (s) \cos \left(\frac{1}{2} s\right)-\frac{1}{4} \sin \left(\frac{9}{2} s\right) \sin (s) \cos \left(\frac{1}{2} s\right)\right. \\
& \left.-4 \cos \left(\frac{9}{2} s\right) \sin (s) \sin \left(\frac{1}{2} s\right)-\sin \left(\frac{9}{2} s\right) \cos (s) \sin \left(\frac{1}{2} s\right)\right\} \\
& +s\left\{5 \cos \left(\frac{9}{2} s\right) \cos (s) \sin \left(\frac{1}{2} s\right)-\frac{3}{4} \sin \left(\frac{9}{2} s\right) \sin (s) \sin \left(\frac{1}{2} s\right)\right. \\
& \left.+\frac{9}{2} \cos \left(\frac{9}{2} s\right) \sin (s) \cos \left(\frac{1}{2} s\right)+\sin \left(\frac{9}{2} s\right) \cos (s) \cos \left(\frac{1}{2} s\right)\right\}
\end{aligned}
$$

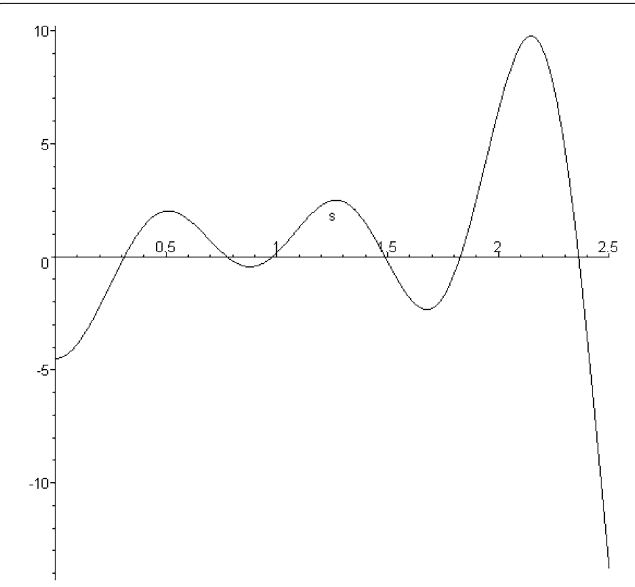

The first five eigenvalues of the example for $\theta=3$ and $\varepsilon=1 / 4$

\begin{tabular}{lcc}
\hline Index & $s$ value & $\lambda$ eigenvalue \\
\hline 1. & 0.3110225636 & 0.09673503507 \\
2. & 0.7732373959 & 0.59789607042 \\
3. & 0.9811690591 & 0.96269272254 \\
4. & 1.487288784 & 2.21202792701 \\
5. & 1.828959916 & 3.34509437433 \\
\hline
\end{tabular}

Figure 5 The graph of the characteristic function for $\theta=3$ and $\varepsilon=1 / 4$. 


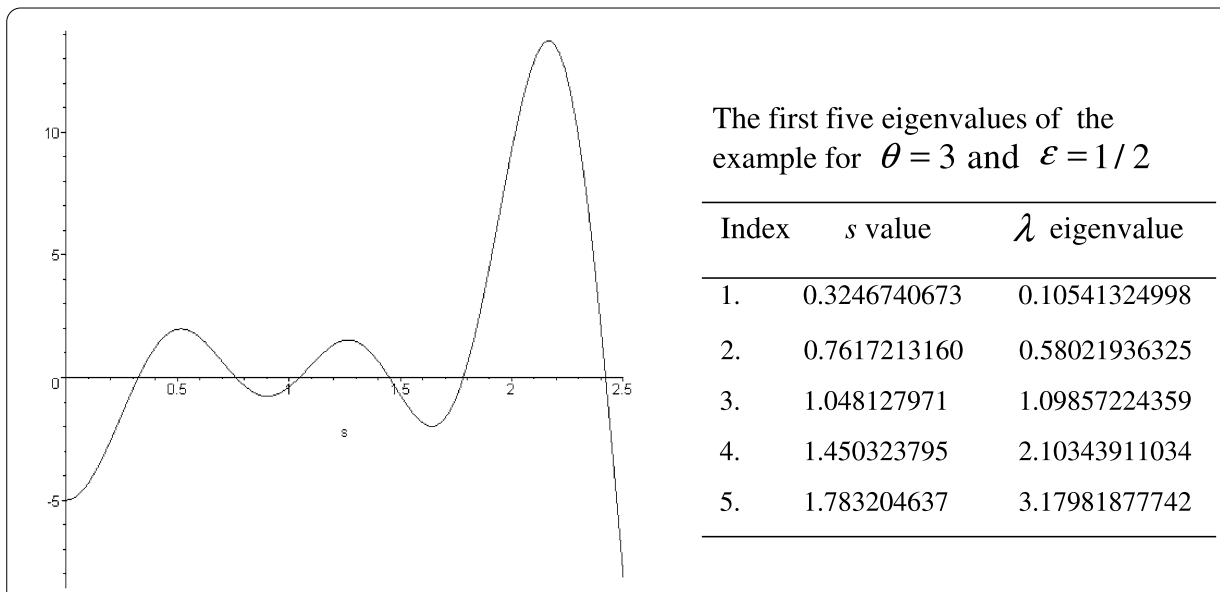

Figure 6 The graph of the characteristic function for $\theta=3$ and $\varepsilon=1 / 2$.

$$
\begin{aligned}
& +\frac{3}{2} \cos \left(\frac{9}{2} s\right) \sin (s) \sin \left(\frac{1}{2} s\right)-4 \cos \left(\frac{9}{2} s\right) \cos (s) \cos \left(\frac{1}{2} s\right) \\
& +\frac{1}{2} \sin \left(\frac{9}{2} s\right) \sin (s) \cos \left(\frac{1}{2} s\right)-\frac{1}{s} \cos \left(\frac{9}{2} s\right) \sin (s) \cos \left(\frac{1}{2} s\right) \\
& =0 .
\end{aligned}
$$

The graph of the characteristic function for $\varepsilon=1 / 2$ is displayed in Figure 6 .

\section{Competing interests}

The authors declare that they have no competing interests.

\section{Authors' contributions}

All authors contributed equally to the writing of this paper. All authors read and approved the final manuscript.

\section{Author details}

'Department of Mathematics, Faculty of Arts and Sciences, Hitit University, Çorum, 19030, Turkey. ${ }^{2}$ Department of Mathematics, Faculty of Arts and Sciences, Ondokuz Mayıs University, Samsun, 55139, Turkey.

\section{Acknowledgements}

All authors are very grateful to the anonymous referees for their valuable suggestions. This paper was supported as a scientific research project (PYO.FEN.1904.11.010) by Ondokuz Mayıs University.

Received: 8 October 2014 Accepted: 1 December 2015 Published online: 16 December 2015

\section{References}

1. Buschmann, D, Stolz, G, Weidmann, J: One-dimensional Schrödinger operators with local point interactions. J. Reine Angew. Math. 467, 169-186 (1995)

2. Titeux, I, Yakubov, Y: Completeness of root functions for thermal conduction in a strip with piecewise continuous coefficients. Math. Models Methods Appl. Sci. 7(7), 1035-1050 (1997)

3. Khmelnytskaya, KV, Serroukh, I: The heat transfer problem for inhomogeneous material in photoacoustic applications and spectral parameter power series. Math. Methods Appl. Sci. (2013). doi:10.1002/mma.2732

4. Tittle, CW: Boundary value problems in composite media. J. Appl. Phys. 36, 1486-1488 (1965)

5. Mulholland, GP, Cobble, MH: Diffusion through composite media. Int. J. Heat Mass Transf. 15(1), 147-160 (1972)

6. Özisik, MN: Heat Conduction. Wiley, New York (1980)

7. Oturanç, G, Sahin, AZ: Eigenvalue analysis of temperature distribution in composite walls. Int. J. Energy Res. 25, 1189-1196 (2001)

8. Hinton, BD: An expansion theorem for an eigenvalue problem with eigenvalue parameter in the boundary conditions. Q. J. Math. 30, 33-42 (1979)

9. Fulton, CT: Two-point boundary value problems with eigenvalues parameter contained in the boundary conditions. Proc. R. Soc. Edinb. A 77A, 293-308 (1977)

10. Walter, J: Regular eigenvalue problems with eigenvalue parameter in the boundary conditions. Math. Z. 133, 301-312 (1973) 
11. Willis, C: Inverse Sturm Liouville problems with two discontinuities. Inverse Probl. 1, $263-289$ (1985)

12. Kobayashi, M: Eigenfunction expansions: a discontinuous version. SIAM J. Appl. Math. 50(3), 910-917 (1990)

13. Altııışı, N, Kadakal, M, Mukhtarov, OS: Eigenvalues and eigenfunctions of discontinuous Sturm Liouville problems with eigenparameter dependent boundary conditions. Acta Math. Hung. 102, 159-175 (2004)

14. Mukhtarov, OS, Kadakal, M: Some spectral properties of one Sturm-Liouville type problem with discontinuous weight. Sib. Math. Zh. 46(4), 860-875 (2004) (in Russian). Translation in Sib. Math. J. 46(4) 681-694 (2005)

15. Kadakal, M, Mukhtarov, OS: Sturm-Liouville problems with discontinuities at two points. Comput. Math. Appl. 54, 1367-1379 (2007)

16. Akdoğan, Z, Demirci, M, Mukhtarov, OS: Green function of discontinuous boundary value problem with transmission conditions. Math. Models Methods Appl. Sci. 30, 1719-1738 (2007)

17. Wang, A, Sun, J, Hao, X, Yao, S: Completeness of eigenfunctions of Sturm Liouville problems with transmission conditions. Methods Appl. Anal. 16(3), 299-312 (2009)

18. Hıra, F, Altınışık, N: Sturm Liouville problem with discontinuity conditions at several points. AIP Conf. Proc. 1470, 126-129 (2012)

19. Tharwat, MM, Bhrawy, AH, Alofi, AS: Approximation of eigenvalues of discontinuous Sturm-Liouville problems with eigenparameter in all boundary conditions. Bound. Value Probl. (2013). doi:10.1186/1687-2770-2013-132

20. Titchmarsh, EC: Eigenfunctions Expansion Associated with Second Order Differential Equations I, 2nd edn. Oxford University Press, London (1962)

\section{Submit your manuscript to a SpringerOpen ${ }^{\mathcal{O}}$ journal and benefit from:}

- Convenient online submission

- Rigorous peer review

- Immediate publication on acceptance

- Open access: articles freely available online

- High visibility within the field

- Retaining the copyright to your article 